\title{
Isomorphic and isometric structure of the optimal domains for Hardy-type operators
}

\author{
by \\ Tomasz Kiwerski (Poznań), Pawee Kolwicz (Poznań) and \\ Lech Maligranda (Luleå and Poznań)
}

\begin{abstract}
We investigate the structure of optimal domains for the Hardy-type operators including, for example, the classical Cesàro, Copson and Volterra operators as well as some of their generalizations. We prove that, in some sense, the abstract Cesàro and Copson function spaces are closely related to the space $L^{1}$, namely, they contain "in the middle" a complemented copy of $L^{1}[0,1]$ and an asymptotically isometric copy of $\ell^{1}$, and can also be renormed to contain an isometric copy of $L^{1}[0,1]$. Moreover, generalized Tandori function spaces are quite similar to $L^{\infty}$ because they contain an isometric copy of $\ell^{\infty}$ and can be renormed to contain an isometric copy of $L^{\infty}[0,1]$. Several applications to the metric fixed point theory will be given. Next, we prove that the Cesàro construction $X \mapsto C X$ does not commute with the truncation operation of the measure space support. We also study whether a given property transfers between a Banach function space $X$ and the space $T X$, where $T$ is the Cesàro or the Copson operator. In particular, we find a large class of properties which do not lift from $T X$ into $X$ and we prove that abstract Cesàro and Copson function spaces are never reflexive, are not isomorphic to a dual space and do not have the Radon-Nikodym property in general.
\end{abstract}

1. Introduction. In 1925 G. H. Hardy [35] proved the following inequality, which today is usually called the classical Hardy inequality:

$$
\int_{0}^{\infty}\left(\frac{1}{x} \int_{0}^{x} f(t) \mathrm{d} t\right)^{p} \mathrm{~d} x \leq\left(\frac{p}{p-1}\right)^{p} \int_{0}^{\infty} f(x)^{p} \mathrm{~d} x,
$$

where $1<p<\infty$ and $f$ is a nonnegative real-valued Lebesgue measurable function (see [36, p. 240] and [53, Chapter 3] for more details). This inequality

2020 Mathematics Subject Classification: Primary 46E30; Secondary 46B20, 46B42.

Key words and phrases: Banach function spaces, symmetric spaces, Cesàro, Copson, Volterra and Tadori function spaces, Cesàro (Hardy), Copson and Volterra operators, (weak) fixed point property, asymptotically isometric copy of $\ell^{1}$, complemented subspaces, renorming, dual Banach space, Radon-Nikodym property.

Received 11 February 2020; revised 1 August 2020.

Published online 18 February 2021. 
can be reformulated in the following way:

the Hardy operator $f \mapsto \frac{1}{x} \int_{0}^{x} f(t) \mathrm{d} t$ maps $L^{p}$ continuously into itself.

Given an operator $T \in \mathcal{L}(Y, X)$, where $X$ and $Y$ are Banach function spaces, it is natural to ask whether there is a Banach function space, say $Z$, such that $T: Z \rightarrow X$ is also bounded and $Z$ is the largest, in the sense of inclusion, Banach function space with this property. This situation can be summarized by the diagram

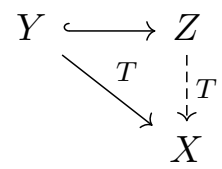

Under some technical assumptions [21, p. 196], $Z$ is the space of all measurable functions $f$ such that $T|f| \in X$, equipped with the norm $\|f\|_{Z}=$ $\|T|f|\|_{X}$. In other words, the space $Z$ is the maximal or optimal domain for the operator $T$ considered with values in the fixed space $X$ and throughout this paper we adopt the convention of denoting it by $T X$. This point of view turned out to be helpful and fruitful in the study of such classes of operators like kernel operators (special cases of operators in this class are, for example, the Volterra, Cesàro, Copson, Poisson or Riemann-Liouville operators), differential operators, convolutions, Fourier transform and the Sobolev embedding (see [65] and references given there).

The classical Cesàro and Copson function spaces appeared in a natural way as the optimal domains of the Hardy operator and its conjugate operator, respectively (see [22]- 25], [54, 64] and [68]). For this reason and also to avoid the use of the term "Hardy space", which is usually reserved for certain spaces of holomorphic functions on the unit disc (interestingly, introduced by F. Riesz in 1923 also to honour Hardy), we will call the above mentioned operator the Cesàro operator $C$, i.e., $(C f)(x):=\frac{1}{x} \int_{0}^{x} f(t) \mathrm{d} t$, remembering Cesàro's result on uniform convergence of averages of partial sums of Fourier series. There is also a connection between the Cesàro function spaces $C X$ and the so-called down spaces $X^{\downarrow}$ introduced by Sinnamon. Namely, for a symmetric space $X$ on $I=[0, \infty)$ such that the Cesàro operator $C$ is bounded on $X$ we can identify $C X$ with $X^{\downarrow}$ (see [70]-[74]; see also [33] and [54, Section 3] for some additional remarks).

From the isomorphic point of view, the abstract Cesàro function spaces $C X$ as well as the abstract Copson function spaces $C^{*} X$ are a kind of a nontrivial mixture of the Banach function space $X$ and $L^{1}$ in which the properties of both spaces manifest themselves. Following this idea, we will look for "the best possible" copies of $\ell^{1}$ and $L^{1}[0,1]$ in the Cesàro and Cop- 
son function spaces, and also of the space $\ell^{\infty}$ in $\operatorname{Ces}_{\infty}:=C L^{\infty}$. We apply our results, e.g., to fixed point theory, a wide branch of functional analysis that has been extensively developed for several decades (see [34] and [44]). It has many applications, for example, in nonlinear analysis as well as in integral and differential equations. In particular, the question whether a Banach space $X$ has or fails the (weak) fixed point property for nonexpansive mappings is fundamental in this area.

In [6, Theorems 1 and 2] Astashkin-Maligranda proved that the Cesàro function spaces $\operatorname{Ces}_{p}:=C L^{p}$ for $1 \leq p \leq \infty$ if $I=[0,1]$ and $1<p \leq \infty$ if $I=[0, \infty)$ fail to have the fixed point property for nonexpansive mappings. In contrast, it was proved by Cui-Hudzik [18], Cui-Hudzik-Li [19], and CuiMeng-Płuciennik [20] that their sequence counterparts, i.e., the Cesàro sequence spaces $\operatorname{ces}_{p}:=C \ell^{p}$, have this property whenever $1<p<\infty$. We will show that the abstract Cesàro and Copson function spaces on two separable measure spaces $[0,1]$ and $[0, \infty)$ contain an order asymptotically isometric copy of $\ell^{1}$ (the notions of asymptotic isometries are intermediate between the isomorphic and isometric theory) and thus, by the Dowling-Lennard result, fail to have the fixed point property in general. In the case of Cesàro function spaces this result can be seen as an essential generalization of the AstashkinMaligranda result from [6]. In fact, the main idea to find an asymptotically isometric copy of $\ell^{1}$ (which, by the way, were introduced precisely to show that certain spaces fail to have the fixed point property) remains the same but our argument is much more sophisticated and works in full generality. An analogous result for the Copson function spaces is new even for $X=L^{p}$. On the other hand, we also prove that nontrivial Tandori function spaces $\widetilde{X}$ contain an order isomorphically isometric copy of $\ell^{\infty}$ and consequently fail to have even the weak fixed point property.

The second important problem we consider is whether "some" property can be transfered from a simpler structure to more complicated one and vice versa. This type of problems has been successfully considered for many constructions. For example, we can mention three of such questions: $1^{\circ}(X, E) \mapsto E(X)$, where $X$ is a Banach space, $E$ is a Banach function space and $E(X)$ is a Köthe-Bochner space, $2^{\circ}(X, Y) \mapsto \mathfrak{F}(X, Y)$, where $X$ and $Y$ are symmetric spaces and $\mathfrak{F}$ is an interpolation functor (see references in [56]), and $3^{\circ} X \mapsto X^{(*)}$, where $X$ is a Banach function space and $X^{(*)}$ is the symmetrization of $X$ (see references in [49]).

We will also consider this problem but for the Cesàro and Copson construction $X \mapsto T X$ presenting a large class of properties that never transfer from $T X$ to $X$. We also give some positive results in this area.

Finally, we will examine the Cesàro construction $X \mapsto C X$ itself. More precisely, we show that this construction does not commute in general with 
the truncation operation $\left.X \mapsto X\right|_{[0,1]}$ highlighting in this way the difference between the Cesàro function spaces defined on $[0,1]$ and $[0, \infty)$. The question whether two operations commute has been often investigated. For example, the symmetrization operation $X \mapsto X^{(*)}$ commutes with the CalderónLozanovskil construction $\rho(X, Y)$ (in particular with the pointwise product $X \odot Y$ ) and with the pointwise multipliers $M(X, Y)$ (in particular with the Köthe dual $X^{\prime}$ ) - see [49]. Furthermore, the Cesàro construction $X \mapsto C X$ commutes with the interpolation functor $\mathfrak{F}$ having the homogeneity property (see [56, Theorem 6]).

It is worth mentioning that we are able to prove most of the results without the assumption that the Cesàro operator or the Copson operator is bounded on $X$, the assumption which is present in almost all previous results of this type.

The paper is organized as follows. After an introduction we collect some necessary definitions, basic facts and notations in Section 2. Here we also recall the duality theorem of Leśnik-Maligranda [54], the LindenstraussTzafriri [57] and Boyd [16] results on interpolation because we will use them frequently.

In Section 3 we will provide some basic results regarding nontriviality of abstract Copson function spaces (Lemma 3.1 and Corollary 3.2). We also discuss the difference between the condition $T X \neq\{0\}$ and the fact that the operator $T$ is bounded on $X$, where $T$ is the Cesàro or the Copson operator (Example 3.3).

Section 4 starts with two lemmas 4.1 and 4.2 which will play a crucial role later on. In particular, they show that the nontrivial Cesàro and Copson function spaces contain "in the middle" a complemented copy of $L^{1}[0,1]$. Next, we prove that a Banach space $X$ which contains a complemented copy of a space $Y$ can always be renormed to contain an isometric copy of $Y$ (Theorem 4.4). As a corollary we deduce immediately that Cesàro and Copson function spaces can be renormed to contain an isometric copy of $L^{1}[0,1]$ and that Tandori function spaces can be renormed to contain an isometric copy of $L^{\infty}[0,1]$. Finally, in Theorems 4.5 and 4.6 we present the main result of this section: Cesàro and Copson function spaces always contain an order asymptotically isometric copy of $\ell^{1}$. Since generalized Tandori function spaces $\widetilde{X}$ and the space $\mathrm{Ces}_{\infty}$ are never order continuous [54, Theorem 1(e)] it follows that they contain an isomorphic copy of $\ell^{\infty}$. Nevertheless, we prove that $\widetilde{X}$ and $\mathrm{Ces}_{\infty}$ always contain an order isometric copy of $\ell^{\infty}$ (Propositions 4.8 and 4.9 .

Next, in Section 5, we try to compare Cesàro function spaces defined on $[0,1]$ and on $[0, \infty)$ and we show that the Cesàro construction $X \mapsto C X$ and the truncation operation of the measure space support $\left.X \mapsto X\right|_{[0,1]}$ do 
not commute in general (Lemma 5.1). This fact explains, in a sense, quite surprising differences between some results obtained for the Cesàro function spaces on a finite and infinite interval (see also [7], [54] and [56]).

In Section 6, we analyze the problem of transferring properties between $X$ and $T X$, where $T=C$ or $T=C^{*}$. We give examples of properties which lift from a Banach function space $X$ to $T X$ and vice versa (Corollary 6.1 and Lemma 6.3. Next, using the results of Bessaga-Pełczyński and Talagrand we find that Cesàro and Copson function spaces are not isomorphic to a dual space and do not have the Radon-Nikodym property (Corollary 6.4). Moreover, we include an example of a certain class of Banach function spaces which contain "in the middle" an isomorphic copy of a Banach function space $Y$, but the construction $X \mapsto T X$, in a sense, forgets about this copy (Lemma 6.2). The presented comparison of this example with the result from [4] can be instructive. Furthermore, we give a large class of properties (including, for example, order continuity, $p$-concavity and the Dunford-Pettis property) which do not transfer from $T X$ to $X$ (Theorem 6.6).

The main result in Section 7 is Theorem 7.1, which states that abstract Cesàro and Copson function spaces fail to have the fixed point property in general. We prove that, under additional assumptions, these spaces cannot even be renormed to have the fixed point property (Corollary 7.4). We also conclude that generalized Tandori function spaces $\widetilde{X}$ and the space $\operatorname{Ces}_{\infty}$ fail to have the weak fixed point property (Proposition 7.5).

Section 8 presents a certain way of generalizing the results from the previous sections. We show that the methods developed in Sections 4 and 7 also work for a wider class of operators, e.g., for the weighted Cesàro operator $\mathcal{H}_{w}$ and its conjugate $\mathcal{H}_{w}^{*}$ (Theorems 8.2 and 8.3 . In particular, we prove that an abstract Volterra space $V X$ fails to have the fixed point property as well (Corollary 8.4.

Finally, the Appendix is devoted to the analysis of the functions $F_{X}$ and $G_{X}$ that appeared in the proof of Theorems 4.5 and 4.6 . We finish this section with a few examples (Example 9.3). In the first one we give some rather exotic examples of the functions $F_{X}$ and in the next one we justify that the order continuity of a symmetric space $X$ is not crucial for the continuity of $F_{X}$.

\section{Notation and preliminaries}

2.1. Banach function spaces and symmetric spaces. Denote by $m$ the Lebesgue measure on $I$, where $I=[0,1]$ or $I=[0, \infty)$, and by $L^{0}=$ $L^{0}(I)$ the set of all equivalence classes of real-valued Lebesgue measurable functions defined on $I$. A Banach function space (or a Banach ideal space) $X=\left(X,\|\cdot\|_{X}\right)$ on $I$ is understood to be a Banach space $X$ such that $X$ is 
a linear subspace of $L^{0}(I)$ satisfying the ideal property: if $f, g \in L^{0}(I)$ with $|f(t)| \leq|g(t)|$ for almost all $t \in I$, and $g \in X$, then $f \in X$ and $\|f\|_{X} \leq\|g\|_{X}$. Unless otherwise stated we assume that a Banach function space $X$ contains a function $f_{0} \in X$ which is positive almost everywhere on $I$ (such a function is called the weak unit in $X$ ), which means that $\operatorname{supp}(X)=I$. Sometimes we will write $X[0,1]$ or $X[0, \infty)$ to stress that the Banach function space $X$ is defined on $I=[0,1]$ or on $I=[0, \infty)$. We say that a Banach function space $X$ is nontrivial if $X \neq\{0\}$.

For two Banach function spaces $X$ and $Y$ on $I$, the symbol $X \stackrel{M}{\longleftrightarrow} Y$ signifies that the inclusion $X \subset Y$ is continuous with norm no greater than $M$, i.e., there exists a constant $M>0$ (the embedding constant) such that $\|f\|_{Y} \leq M\|f\|_{X}$ for all $f \in X$. If the embedding $X \stackrel{M}{\hookrightarrow} Y$ holds with some (maybe unknown) constant $M>0$ we simply write $X \hookrightarrow Y$ and $\|f\|_{Y} \lesssim\|f\|_{X}$. Recall also that for two Banach function spaces $X$ and $Y$ the inclusion $X \subset Y$ is always continuous. Moreover, $X=Y$ (resp. $X \equiv Y$ ) means that the spaces $X$ and $Y$ have the same elements and their norms are equivalent (resp. equal). If the spaces $X$ and $Y$ are isomorphic (resp. are isometric under the isometry $\lambda \cdot \mathrm{id}$, where $\lambda>0$ ), then we write $X \simeq Y$ (resp. $X \cong Y$ ).

Recall that the Köthe dual space (or associated space) $X^{\prime}=X^{\prime}(I)$ of a Banach function space $X$ on $I$ is defined as

$$
X^{\prime}:=\left\{f \in L^{0}(I):\|f\|_{X^{\prime}}=\sup _{g \in X,\|g\|_{X} \leq 1} \int_{I}|f(x) g(x)| \mathrm{d} x<\infty\right\} .
$$

The Köthe dual space is again a Banach function space. Moreover, $X \stackrel{1}{\hookrightarrow} X^{\prime \prime}$ $:=\left(X^{\prime}\right)^{\prime}$, and $X=X^{\prime \prime}$ if and only if the norm in $X$ has the Fatou property (written $X \in(\mathrm{FP})$ ), i.e., for any sequence $\left(f_{n}\right) \subset X$ with $0<f_{n} \uparrow f$ almost everywhere on $I$ such that $\sup _{n \in \mathbb{N}}\left\|f_{n}\right\|_{X}<\infty$, we have $f \in X$ and $\left\|f_{n}\right\|_{X} \uparrow\|f\|_{X}$.

A function $f \in X$, where $X$ is a Banach function space space on $I$, is said to have order continuous norm in $X$ if for any decreasing sequence of sets $A_{n} \subset I$ with empty intersection, we have $\left\|f \chi_{A_{n}}\right\|_{X} \rightarrow 0$ as $n \rightarrow \infty$ (see [14. Proposition 3.5, p. 15]). We denote by $X_{a}$ the subspace of all functions with order continuous norm in $X$. A Banach function space $X$ on $I$ is order continuous (we write $X \in(\mathrm{OC})$ ) if every element of $X$ has order continuous norm, that is, $X_{a}=X$. The subspace $X_{a}$ is always closed in $X$ [14, Theorem 3.8, p. 16]. If $X$ is an order continuous Banach function space then $X^{*}=X^{\prime}[14$, Theorem 4.1, p. 20]. Moreover, a Banach function space on $I$ with the Fatou property is reflexive if and only if both $X$ and $X^{\prime}$ are order continuous (cf. [14, Corollary 4.4, p. 23]).

Throughout the paper, whenever we take a subset $A \subset I$, we mean that $A$ is Lebesgue measurable. For a function $f \in L^{0}(I)$ we define the 
support of $f$ as

$$
\operatorname{supp}(f):=\{x \in I: f(x) \neq 0\} .
$$

For a measurable function $w: I \rightarrow(0, \infty)$ (a weight on $I$ ) and for a Banach function space $X$ on $I$, the weighted Banach function space $X(w)=$ $X(w)(I)$ is defined as

$$
X(w):=\left\{f \in L^{0}(I): f w \in X\right\},
$$

with the norm $\|f\|_{X(w)}=\|f w\|_{X}$. It is clear that $X(w)$ is a Banach function space on $I$ and $X(w)^{\prime} \equiv X^{\prime}(1 / w)$.

For a function $f \in L^{0}(I)$ we define the distribution function $d_{f}(\lambda):=$ $m(\{t \in I:|f(t)|>\lambda\})$ for $\lambda>0$. We say that functions $f, g \in L^{0}(I)$ are equimeasurable if they have the same distribution functions, i.e. $d_{f} \equiv d_{g}$. By a symmetric space (symmetric Banach function space or rearrangement invariant Banach function space) on $I$ we mean a Banach function space $E=\left(E,\|\cdot\|_{E}\right)$ on $I$ with the additional property that for any equimeasurable functions $f, g \in L^{0}(I)$ if $f \in E$ then $g \in E$ and $\|f\|_{E}=\|g\|_{E}$. In particular, $\|f\|_{E}=\left\|f^{*}\right\|_{E}$, where $f^{*}(t):=\inf \left\{\lambda>0: d_{f}(\lambda) \leq t\right\}$ for $t \geq 0$.

For general properties of Banach lattices, Banach function spaces and symmetric spaces we refer to the books by Bennett-Sharpley [14], Kantorovich-Akilov [43], Krein-Petunin-Semenov [51], Lindenstrauss-Tzafriri [57], Maligranda [61], Meyer-Nieberg [63], and Wnuk [77].

2.2. Cesàro, Copson and Tandori function spaces. For a Banach function space $X$ on $I$ the abstract Cesàro function space $C X=C X(I)$ is defined as

$$
C X:=\left\{f \in L^{0}(I): C|f| \in X\right\} \quad \text { with the norm } \quad\|f\|_{C X}:=\|C \mid f\|_{X},
$$

where $C$ denotes the Cesàro operator (sometimes also called the Hardy operator)

$$
C: f \mapsto C f(x):=\frac{1}{x} \int_{0}^{x} f(t) \mathrm{d} t \quad \text { for } 0<x \in I .
$$

Copson and Tandori spaces are directly related to Cesàro spaces. For a Banach ideal space $X$ on $I$ we define the abstract Copson function space $C^{*} X=C^{*} X(I)$ as

$$
C^{*} X:=\left\{f \in L^{0}(I): C^{*}|f| \in X\right\} \quad \text { with the norm } \quad\|f\|_{C^{*} X}:=\left\|C^{*}|f|\right\|_{X},
$$

where $C^{*}$ denotes the conjugate operator (in the sense of Köthe) to the Cesàro operator $C$, which will be called the Copson operator:

$$
C^{*}: f \mapsto C^{*} f(x):=\int_{I \cap[x, \infty)} \frac{f(t)}{t} \mathrm{~d} t \quad \text { for } x \in I,
$$


and the abstract Tandori function space $\widetilde{X}=\widetilde{X}(I)$ is

$$
\widetilde{X}:=\left\{f \in L^{0}(I): \widetilde{f} \in X\right\} \quad \text { with the norm } \quad\|f\|_{\widetilde{X}}:=\|\widetilde{f}\|_{X},
$$

where the nonincreasing majorant $\tilde{f}$ of a function $f$ is defined by

$$
\widetilde{f}(x):=\operatorname{esssup}_{t \in I, t \geq x}|f(t)| \quad \text { for } x \in I .
$$

In the sequence case, the discrete Cesàro and Copson operators $C_{d}$ and $C_{d}^{*}$ are defined for $n \in \mathbb{N}$ by

$$
\left(C_{d} a\right)_{n}:=\frac{1}{n} \sum_{k=1}^{n} a_{k} \quad \text { and } \quad\left(C_{d}^{*} a\right)_{n}:=\sum_{k=n}^{\infty} \frac{a_{k}}{k},
$$

respectively. Moreover, the nonincreasing majorant $\widetilde{a}=\left(\widetilde{a}_{n}\right)$ of a sequence $a=\left(a_{n}\right)$ is defined as $\widetilde{a}_{n}:=\sup _{k \in \mathbb{N}, k \geq n}\left|a_{k}\right|$ for $n \in \mathbb{N}$. Then the corresponding abstract Cesàro sequence space $C X$, abstract Copson sequence space $C^{*} X$ and abstract Tandori sequence space $\widetilde{X}$ are defined analogously to 2.1$)-2.3$.

Abstract Cesàro function spaces are a generalization of the well-known classical Cesàro spaces $\operatorname{Ces}_{p}[0,1]$ and $\operatorname{Ces}_{p}[0, \infty)$. Indeed, if we take $X=L^{p}$, where $1 \leq p \leq \infty$, then $\operatorname{Ces}_{p}=C L^{p}$ (note that for $p=1$ we have $\operatorname{Ces}_{1}[0,1]=$ $L^{1}(\ln (1 / t))$ and $\left.\operatorname{Ces}_{1}[0, \infty)=\{0\}\right)$. The space $\operatorname{Ces}_{\infty}[0,1]$ already appeared in 1948 and it is known as the Korenblyum-Krein-Levin space $K$ (see [50], [77, p. 26 and p. 61] and [78, pp. 469-471]).

Various properties of these spaces have been studied by Astashkin [4], Astashkin-Maligranda [6]-[12], Hassard-Hussein [37], Kamińska-Kubiak [41], Kubiak [52], Shiue [69] and Sy-Zhang-Lee [75]. Taking $X=L^{\Phi}, X=\Lambda_{\varphi}$ or $X=M_{\varphi}$ we obtain Cesàro-Orlicz, Cesàro-Lorentz and CesàroMarcinkiewicz spaces, respectively, which have been intensively studied by Astashkin-Leśnik-Maligranda [5], Kiwerski-Kolwicz [45]-[47], and KiwerskiTomaszewski [48]. A general discussion of this construction when $X$ is a Banach function space or a symmetric space was initiated in [54] and [55]. More recently, the structure of these spaces, especially in their general form, is quite popular among researchers, including Astashkin-Leśnik-Maligranda [5], Curbera-Ricker [22], Delgado-Soria [25], and Kiwerski-Tomaszewski [48].

Note that Cesàro function spaces $C X$ are never symmetric or reflexive. Nevertheless, at least when $X$ is a symmetric space, there are some connections and similarities to the classical theory of normed ideal spaces and symmetric spaces. For example, it has been shown in [48, Theorem 3] that order continuity "transfers" quite well between $X$ and $C X$. Moreover, Ces $\infty$ and $\operatorname{ces}_{\infty}$ are isomorphic [5, Theorem 13] (this is analogous to Pełczyński's well-known result [66] that $L^{\infty}$ and $\ell^{\infty}$ are isomorphic). Furthermore, $\widetilde{\ell^{1}}$ has the Schur property but is not isomorphic to $\ell^{1}$ [5, Theorem 3.1]. Of course, 
there are also major differences between the cases of a finite and infinite interval, for example in results on the Köthe duality for abstract Cesàro function spaces [54, Theorems 3-5] (cf. also Theorem A below) or in the interpolation results proved in [56].

It is worth mentioning here that the study of the classical Cesàro sequence spaces $\operatorname{ces}_{p}=C \ell^{p}$ for $1<p \leq \infty$ began much earlier and many results have been obtained: see [7], [12], [13] and [40] and the references therein.

The Copson function spaces $\operatorname{Cop}_{p}=C^{*} L^{p}$ and Copson sequence spaces $\operatorname{cop}_{p}=C^{*} \ell^{p}$ have already appeared in Bennett's memoir [13, pp. 25-28 and p. 123]. Furthermore, Astashkin-Maligranda [9, Section 2] used $\operatorname{Cop}_{p}$ in their interpolation results. Abstract Copson spaces have been studied by Leśnik-Maligranda [56]. For some connections between Cesàro and Copson function spaces and their iterations $C C X$ and $C^{*} C^{*} X$ we refer to [56, Theorem $1(\mathrm{a}, \mathrm{b})]$.

Leśnik and Maligranda [54] suggested calling $\widetilde{X}$ a generalized Tandori space since Tandori [76] proved in 1954 that $\left(\operatorname{Ces}_{\infty}[0,1]\right)^{\prime}=\widetilde{L^{1}}[0,1]$. Moreover, these spaces appeared earlier but without such name, e.g., in [7] and [55]. Tandori spaces are related to the Köthe duality of Cesàro spaces. Many special cases of this general construction have been studied by Alexiewicz [2], Astashkin-Maligranda [7], Bennett [13], Jagers [39], KamińskaKubiak [40]-41] and Luxemburg-Zaanen [59]. General Tandori spaces $\widetilde{X}$ have been studied by Leśnik-Maligranda [54]-[56] and the following Köthe duality result has been proved in [54, Theorems 3,5 and 6].

THeOrem A. If $X$ is a Banach function space on $I=[0, \infty)$ such that the Cesàro operator $C$ and the dilation operator $\sigma_{\tau}$ (for some $0<\tau<1$ ) are bounded on $X$, then

$$
(C X)^{\prime}=\widetilde{X^{\prime}}
$$

Furthermore, if $X$ is a symmetric space on $I=[0,1]$ with the Fatou property such that both $C$ and $C^{*}$ are bounded on $X$, then

$$
(C X)^{\prime}=\widehat{X^{\prime}(w)} \text { where } w:[0,1) \ni x \mapsto \frac{1}{1-x} .
$$

The dilation operator $\sigma_{\tau}$ for $\tau>0$ is defined by $\sigma_{\tau} f(x):=f(x / \tau)$ for $0<x<\infty$ and

$$
\sigma_{\tau} f(x):= \begin{cases}f(x / \tau) & \text { if } x<\min \{1, \tau\} \\ 0 & \text { if } \tau \leq x<1,\end{cases}
$$

for $0<x \leq 1$. This operator is bounded in any symmetric space $X$ on $I$ and $\left\|\sigma_{\tau}\right\|_{X \rightarrow X} \leq \max \{1, \tau\}$ (see [14, p. 148] and [51, pp. 96-98]). The Boyd 
indices of a symmetric space $X$ are defined by

$$
p(X):=\lim _{\tau \rightarrow \infty} \frac{\ln \tau}{\ln \left\|\sigma_{\tau}\right\|_{X \rightarrow X}} \quad \text { and } \quad q(X):=\lim _{\tau \rightarrow 0^{+}} \frac{\ln \tau}{\ln \left\|\sigma_{\tau}\right\|_{X \rightarrow X}} .
$$

These numbers may be different for the same $X$ on $I=[0,1]$ and on $I=$ $[0, \infty)$, but always $1 \leq p(X) \leq q(X) \leq \infty$ (see [51], [57] and [60]).

We will use the following result from Lindenstrauss-Tzafriri's book [57, Proposition 2.b.3, p. 132].

TheOREM B. If $X$ is a symmetric space on $I$, then there are constants $A, B>0$ such that

$$
L^{p} \cap L^{q} \stackrel{A}{\hookrightarrow} X \stackrel{B}{\hookrightarrow} L^{p}+L^{q}
$$

for all $p, q>0$ satisfying $1 \leq p<p(X)$ and $q(X)<q \leq \infty$, where $p(X)$ and $q(X)$ are the Boyd indices of $X$, and

$$
\begin{aligned}
& L^{p} \cap L^{q}:=\left\{f \in L^{0}(I):\|f\|_{L^{p} \cap L^{q}}=\max \left\{\|f\|_{L^{p}},\|f\|_{L^{q}}\right\}<\infty\right\}, \\
& L^{p}+L^{q}:=\left\{f \in L^{0}(I):\|f\|_{L^{p}+L^{q}}=\inf _{\substack{f=g+h \\
g \in L^{p}, h \in L^{q}}}\left\{\|g\|_{L^{p}}+\|h\|_{L^{q}}\right\}<\infty\right\} .
\end{aligned}
$$

Moreover, if $p(X)=1$ (resp. $q(X)=\infty)$ then we can take $p=1$ (resp. $q=\infty)$ in (2.6).

Let us recall an important result about boundedness of the Cesàro operator (cf. [53, Theorem 17, p. 130]).

Theorem C. Let $X$ be a symmetric space on $I$. Then

(i) the Cesàro operator $C$ is bounded on $X$ if and only if $p(X)>1$,

(ii) the Copson operator $C^{*}$ is bounded on $X$ if and only if $q(X)<\infty$.

Throughout the article we will use the following notation: the norm of the function $f_{\lambda}: I \ni x \mapsto \frac{1}{x} \chi_{[\lambda, m(I))}(x)$, where $0<\lambda \in I$, in a Banach function space $X$ on $I$ will be denoted by $\left\|\frac{1}{x} \chi_{[\lambda, m(I))}(x)\right\|_{X(I)}$, i.e.,

$$
\left\|f_{\lambda}\right\|_{X(I)}:=\left\|\frac{1}{x} \chi_{[\lambda, m(I))}(x)\right\|_{X(I)},
$$

and the norm of the function $(C|f|) \chi_{A}: I \ni x \mapsto \frac{1}{x} \int_{0}^{x}|f(t)| \mathrm{d} t \chi_{A}(x)$, where $A \subset I$, will be denoted by

$$
\left\|\frac{1}{x} \int_{0}^{x}|f(t)| \mathrm{d} t \chi_{A}(x)\right\|_{X(I)}:=\left\|(C|f|) \chi_{A}\right\|_{X(I)} .
$$

Recall that if $X$ is a Banach function space on $I$ and $X \in(\mathrm{FP})$, then the Cesàro operator $C$ is bounded on $X$ if and only if the Copson operator $C^{*}$ is bounded on $X^{\prime}$, and $\|C\|_{X \rightarrow X}=\left\|C^{*}\right\|_{X^{\prime} \rightarrow X^{\prime}}$ [49, Remark 1(iv)]. Note 
also that if $X$ is a Banach function space on $I$, then the assumption that $C$ maps $X$ into $X$ is in fact equivalent to $C$ being bounded on $X$ (48]; alternatively, it is also due to the fact that every positive linear operator acting between Banach lattices is continuous [63, Proposition 1.3.5]). Clearly, if $C$ is bounded on $X$, then $X \hookrightarrow C X$. Therefore, the space $C X$ is nontrivial with $\operatorname{supp}(C X)=\operatorname{supp}(X)=I$.

We will now collect some other useful facts about an abstract Cesàro function space $C X$, which are proved in [5, proof of Proposition 2.2], [54, Theorem 1(a,b)] and [48, Lemma 2].

Theorem D. Let $X$ be a Banach function space on $I$. Then

(i) $C X[0,1]$ is nontrivial if and only if $\chi_{[\lambda, 1]} \in X$ for some $0<\lambda<1$,

(ii) $C X[0, \infty)$ is nontrivial if and only if $\frac{1}{x} \chi_{[\lambda, \infty)}(x) \in X$ for some $\lambda>0$.

In particular, $[\lambda, m(I)) \subset \operatorname{supp}(C X)$ for some $0<\lambda<m(I)$.

If $X$ is a Banach function space on $I$ such that the Cesàro operator $C$ is bounded on $X$ or $X$ is a symmetric space on $[0,1]$ or $X$ is a symmetric space on $[0, \infty)$ with $C X[0, \infty) \neq\{0\}$, then

(iii) $\chi_{[\lambda, 1]} \in X$ for all $0<\lambda<1$ if $I=[0,1]$,

(iv) $\frac{1}{x} \chi_{[\lambda, \infty)}(x) \in X$ for all $\lambda>0$ if $I=[0, \infty)$.

In particular, $\operatorname{supp}(C X)=\operatorname{supp}(X)=I$. If $X$ is a symmetric space on $[0,1]$, then $C X$ is always nontrivial.

3. Some auxiliary results. We give a few simple but useful facts about Copson spaces.

Lemma 3.1. Let $X$ be a Banach function space on I. Then the Copson space $C^{*} X$ is nontrivial if and only if $\chi_{[0, \lambda]} \in X$ for some $0<\lambda<m(I)$.

Proof. Assume that $C^{*} X \neq\{0\}$. Then there exists $f \in C^{*} X$ with $|f(x)|>0$ for $x \in A \subset I$ and $m(A)>0$. Of course, we can also find $\lambda>0$ such that

$$
\int_{\lambda}^{m(I)} \frac{|f(t)|}{t} \mathrm{~d} t=: \eta>0 .
$$

Therefore,

$$
\begin{aligned}
\eta \chi_{[0, \lambda]}(x) & =\int_{\lambda}^{m(I)} \frac{|f(t)|}{t} \mathrm{~d} t \chi_{[0, \lambda]}(x) \leq \int_{x}^{m(I)} \frac{|f(t)|}{t} \mathrm{~d} t \chi_{[0, \lambda]}(x) \\
& \leq C^{*}|f|(x) \in X,
\end{aligned}
$$

so $\chi_{[0, \lambda]} \in X$. 
If $\chi_{[0, \lambda]} \in X$ for some $0<\lambda<m(I)$, then for $0<a<\lambda$ we have

$$
\left\|\chi_{[a, \lambda]}\right\|_{C^{*} X} \leq\left\|\left(\int_{a}^{\lambda} \frac{\mathrm{d} t}{t}\right) \chi_{[0, \lambda]}\right\|_{X}=\ln (\lambda / a)\left\|\chi_{[0, \lambda]}\right\|_{X}<\infty,
$$

which means that $C^{*} X \neq\{0\}$.

\section{Corollary 3.2 .}

(1) The Copson space $C^{*} X$ is always nontrivial whenever $X$ is a symmetric space.

(2) If $X$ is a Banach function space on I such that the operator $C^{*}$ is bounded on $X$, then $\operatorname{supp}\left(C^{*} X\right)=I$. In particular, $C^{*} X$ is nontrivial. Moreover,

(i) $\left.L^{\infty}[0,1]\right|_{[0, \lambda]} \hookrightarrow X[0,1]$ for all $0<\lambda<1$, and in addition $L^{\infty}[0,1] \hookrightarrow$ $X[0,1]$ if $X$ has the Fatou property,

(ii) $L_{\text {fin }}^{\infty}[0, \infty) \subset X[0, \infty)$, and in addition $L_{b}^{\infty}[0, \infty) \hookrightarrow X[0, \infty)$ if $X$ has the Fatou property,

where $L_{\text {fin }}^{\infty}(I):=\left\{f \in L^{\infty}(I): m(\operatorname{supp}(f))<\infty\right\}$ and $\left(L^{\infty}(I)\right)_{b}=$ $L^{\infty}(I)_{b}$ is the closure of $L_{\mathrm{fin}}^{\infty}(I)$ in $L^{\infty}(I)$.

Proof. If $X$ is a symmetric space on $I$, then $\chi_{[0, \lambda]} \in X$ for all $0<\lambda<$ $m(I)$, so $C^{*} X \neq\{0\}$ (see Lemma 3.1).

It is also clear that if $C^{*}$ is bounded on $X$ then $X \hookrightarrow C^{*} X$ and consequently $\operatorname{supp}\left(C^{*} X\right)=I$.

(i) Of course, $\left.L^{\infty}[0,1]\right|_{[0, \lambda]} \hookrightarrow X$ is equivalent to $\chi_{[0, \lambda]} \in X$. Take $0<$ $\lambda<1$ and let $f_{0}$ be a weak unit in $X$. Then $\int_{\lambda}^{m(I)}\left|f_{0}(t)\right| / t \mathrm{~d} t=: \delta>0$ and proceeding as in the proof of Lemma 3.1 we get $\chi_{[0, \lambda]} \in X$. If, additionally, $X \in(\mathrm{FP})$, then $\chi_{[0,1]} \in X$, i.e., $L^{\infty}[0,1] \hookrightarrow X$.

(ii) Similarly to case (i), we find that $L_{\text {fin }}^{\infty}[0, \infty) \subset X[0, \infty)$ (note only that $L_{\text {fin }}^{\infty}$ is not complete, so the inclusion $L_{\text {fin }}^{\infty} \subset L_{b}^{\infty}$ is not continuous). Suppose now that $X \in(\mathrm{FP})$ and take $f \in L_{b}^{\infty}[0, \infty)$. To show that $f \in X$ it is enough to take a sequence $\left(f_{n}\right) \subset L_{\text {fin }}^{\infty}[0, \infty)$ with $0 \leq f_{n} \uparrow f$.

Many results in the theory of Cesàro and Copson function spaces are proved under the assumption that $C$ or $C^{*}$ is bounded on $X$. As mentioned in the introduction, we are able to prove our results under the essentially weaker assumption (actually the weakest possible one) that the Cesàro or Copson function space is nontrivial. In this context, it seems resonable to give several examples discussing the difference between these two assumptions, because many naturally appearing spaces have the property that the operator $T$, where $T=C$ or $T=C^{*}$, is not bounded on $X$ but $T X \neq\{0\}$ or even $\operatorname{supp}(T X)=I$. 
ExAmple 3.3. (a) As in [54, Example 2], consider the space $L^{p}\left(w_{1}\right)$ on $[0, \infty)$, where $1<p<\infty$ and

$$
w_{1}(x)=\frac{1}{1-x} \chi_{[0,1)}(x)+\chi_{[1, \infty)}(x) .
$$

Then $\operatorname{supp}(X)=[0, \infty), \operatorname{supp}(C X)=[1, \infty)$ and $\operatorname{supp}\left(C^{*} X\right)=[0,1]$. Put $X=L^{1}\left(w_{2}\right)$, where

$$
w_{2}: I \ni x \mapsto 1 / x .
$$

Then $\chi_{[0, \lambda]} \notin X$ for every $0<\lambda<m(I)$, so $C^{*} X=\{0\}$. Finally, take $X=L^{\infty}\left(w_{3}\right)$, where

$$
w_{3}=\operatorname{id}_{I}: I \ni x \mapsto x .
$$

Then $C X \equiv L^{1}$. On the other hand, if $C: X \rightarrow X$ is bounded, then $X \hookrightarrow C X$ but $L^{\infty}\left(w_{3}\right) \hookrightarrow L^{1}$ (just take $\left.f(x)=1 / x\right)$, so $C$ is not bounded on $L^{\infty}\left(w_{3}\right)$ and $\operatorname{supp} C X=I$.

(b) It is easy to see that $\operatorname{Ces}_{1}[0,1]$ is just $L^{1}(w)[0,1]$, where $w(t)=\ln (1 / t)$ for $0<t \leq 1$. Indeed,

$$
\int_{0}^{1}\left(\frac{1}{x} \int_{0}^{x}|f(t)| \mathrm{d} t\right) \mathrm{d} x=\int_{0}^{1}\left(\int_{t}^{1} \frac{\mathrm{d} x}{x}\right)|f(t)| \mathrm{d} t=\int_{0}^{1}|f(t)| \ln \left(\frac{1}{t}\right) \mathrm{d} t
$$

(see [7, Theorem 1(a)]). Therefore, although $C$ is not bounded on $L^{1}[0,1]$ (by Theorem $\mathrm{C}$ ), we see again that $\operatorname{supp}\left(\operatorname{Ces}_{1}[0,1]\right)=[0,1]$. Thus, if $f \in$ $\mathrm{Ces}_{1}[0,1]$ and $\operatorname{supp}(f) \subset[a, b]$, where $0<a<b<1$, then

$$
\ln (1 / b)\|f\|_{L^{1}[0,1]} \leq\|f\|_{\operatorname{Ces}_{1}[0,1]} \leq \ln (1 / a)\|f\|_{L^{1}[0,1]}
$$

(see also [6, Lemma 1, inequality (4)]). The equality (3.1) shows by the way that $\operatorname{Ces}_{1}[0, \infty)=\{0\}$ [7, Theorem 1(a)].

(c) Clearly, $\operatorname{Cop}_{1} \equiv L^{1}$ and $\operatorname{Cop}_{\infty} \equiv L^{1}(1 / t)$ because

$$
\|f\|_{\mathrm{Cop}_{1}}=\int_{I}\left(\int_{x}^{m(I)} \frac{|f(t)|}{t} \mathrm{~d} t\right) \mathrm{d} x=\int_{I}\left(\int_{0}^{t} \mathrm{~d} x\right) \frac{|f(t)|}{t} \mathrm{~d} t=\|f\|_{L^{1}},
$$

and

$$
\|f\|_{\mathrm{Cop}_{\infty}}=\sup _{x \in I} \int_{x}^{m(I)} \frac{|f(t)|}{t} \mathrm{~d} t=\int_{0}^{m(I)} \frac{|f(t)|}{t} \mathrm{~d} t=\|f\|_{L^{1}(1 / t)} .
$$

Again, $\operatorname{supp}\left(\operatorname{Cop}_{\infty}\right)=I$ but $C^{*}$ is not bounded on $L^{\infty}$ by Theorem C.

(d) Let $X$ be a symmetric space on $[0,1]$ with $p(X)=1$. Then $C$ is not bounded on $X$ but $C X \neq\{0\}$, by Theorems $C$ and D. For example, if $X$ is the Zygmund space $L \log L[0,1]$ [14, Definition 6.1, p. 243)], then $p(X)=1$ [14, Theorem 6.5, p. 247)].

(e) Consider a symmetric space $X$ on $I$ such that $C^{*}$ is not bounded on $X$. Then, by Lemma 3.1, $C^{*} X \neq\{0\}$, because $\chi_{[0, \lambda]} \in X$ for each $0<\lambda<m(I)$. In particular, we can take $X=L^{\Phi}$, where $L^{\Phi}$ is the Orlicz space generated by 
the Orlicz function $\Phi$ which does not satisfy the $\Delta_{2}$-condition. Since $\Phi \notin \Delta_{2}$, we have $q\left(L^{\Phi}\right)=\infty$ ([57, Proposition 2.5, p. 139] and [60, Theorem 3.2, p. 22]) and consequently $C^{*}$ is not bounded on $X$ by Theorem $\mathrm{C}$.

(f) Suppose that $X$ is the Orlicz space $L^{\Phi}$ generated by

$$
\Phi(x)=x \log (1+x) .
$$

First, note that $p\left(L^{\Phi}\right)=\alpha_{\Phi}$, where $\alpha_{\Phi}$ is the lower Orlicz-Matuszewska index of $\Phi$ [57, Proposition 2.5, p. 139 and Remark 2, p. 140]. Moreover, it is not difficult to calculate that $\alpha_{\Phi}=1$ [60, pp. 7-21]. Consequently, $C$ is not bounded on $X$ by Theorem C. We claim that $C X \neq\{0\}$. This is clear when $I=[0,1]$. In fact, $L^{\Phi}$ is a symmetric space, so $\chi_{[0, \lambda]} \in X$ for each $0<\lambda<1$ and we can apply Theorem D. If $I=[0, \infty)$, according to Theorem $\mathrm{D}$ we need to show that $\left(f_{\lambda}: x \mapsto \frac{1}{x} \chi_{[\lambda, \infty)}(x)\right) \in L^{\Phi}$ for some $\lambda>0$. Recall that $f \in L^{\Phi}$, whenever $\int_{0}^{\infty} \Phi(\gamma|f(x)|) \mathrm{d} x<\infty$ for some $\gamma>0$ (see [61]). We have

$$
\int_{0}^{\infty} \Phi\left(\left|f_{\lambda}(x)\right|\right) \mathrm{d} x=\int_{\lambda}^{\infty} \frac{1}{x} \log \left(1+\frac{1}{x}\right) \mathrm{d} x \leq \int_{\lambda}^{\infty} \frac{1}{x^{2}} \mathrm{~d} x=\frac{1}{\lambda}<\infty,
$$

and the claim follows.

4. Copies of $\ell^{1}, \ell^{\infty}, L^{1}[0,1]$ and $L^{\infty}[0,1]$ in Cesàro, Copson and Tandori function spaces. The norms of Cesàro and Copson function spaces are generated by a positive sublinear operator $T$, where $T$ stands for the Cesàro or the Copson operator, and by the norm of a Banach function space $X$. Thus, in a sense, $T X$ is a nontrivial mix of $L^{1}$ and $X$, and some similarities to both these spaces can be found in $T X$. We will make these statements more precise by showing first that the Cesàro and Copson function spaces contain "good" copies of $L^{1}[0,1]$ and $\ell^{1}$.

Lemma 4.1. Let $X$ be a Banach function space on I such that $C X \neq\{0\}$. Then there are $0<a<b<m(I)$ such that

$$
\left\|\frac{1}{x} \chi_{[b, m(I))}(x)\right\|_{X}\|f\|_{L^{1}(I)} \leq\|f\|_{C X} \leq\left\|\frac{1}{x} \chi_{[a, m(I))}(x)\right\|_{X}\|f\|_{L^{1}(I)}
$$

for all $f \in C X$ with $\operatorname{supp}(f) \subset[a, b]$. In particular, $C X$ contains a complemented copy of $L^{1}[0,1]$.

Proof. We only sketch the proof because this lemma is just a reformulation of [5, Proposition 2.2] (cf. also [12, Theorem 5.1(b)]).

Let $I=[0,1]$. First, $\chi_{[\lambda, 1]} \in X$ for some $0<\lambda<1$ due to nontriviality of $C X$ (see Theorem D). Take $a=\lambda$ and choose $b \in(a, 1)$. Then $\frac{1}{x} \chi_{[a, 1]}(x) \in X$ and, from the ideal property, also $\frac{1}{x} \chi_{[b, 1]}(x) \in X$. Now, for $f \in C X$ with 
$\operatorname{supp}(f) \subset[a, b]$ it is obvious that

$$
\frac{1}{x}\|f\|_{L^{1}[0,1]} \chi_{[b, 1]}(x) \leq \frac{1}{x} \int_{0}^{x}|f(t)| \mathrm{d} t \leq \frac{1}{x}\|f\|_{L^{1}[0,1]} \chi_{[a, 1]}(x)
$$

for any $0<x \in I$. Thus,

$$
\left\|\frac{1}{x} \chi_{[b, 1]}(x)\right\|_{X}\|f\|_{L^{1}[0,1]} \leq\left\|\frac{1}{x} \int_{0}^{x}|f(t)| \mathrm{d} t\right\|_{X} \leq\left\|\frac{1}{x} \chi_{[a, 1]}(x)\right\|_{X}\|f\|_{L^{1}[0,1]}
$$

and (i) follows.

At this point, it is clear that $\left\{f \in L^{1}[0,1]: \operatorname{supp}(f) \subset[a, b]\right\} \simeq L^{1}[0,1]$ and this copy of $L^{1}[0,1]$ is in fact complemented because the projection $P: f \mapsto f \chi_{[a, b]}$ is bounded.

For $I=[0, \infty)$ the proof is completely analogous.

Lemma 4.2. Let $X$ be a Banach function space on $I$ such that $C^{*} X \neq\{0\}$. Then there are numbers $0<a<b<m(I)$ such that

$$
\left\|\chi_{[0, a]}\right\|_{X}\|f\|_{L^{1}(1 / t)(I)} \leq\|f\|_{C^{*} X} \leq\left\|\chi_{[0, b]}\right\|_{X}\|f\|_{L^{1}(1 / t)(I)}
$$

for all $f \in C^{*} X$ with $\operatorname{supp}(f) \subset[a, b]$. In particular, $C^{*} X$ contains a complemented copy of $L^{1}[0,1]$.

Proof. We give the proof for $I=[0,1]$; the other case is analogous.

Suppose $I=[0,1]$. Since $C^{*} X$ is nontrivial we get $\chi_{[0, \lambda]} \in X$ for some $0<\lambda<1$ (see Lemma 3.1). Take $b=\lambda$ and choose $a \in(0, b)$. If $f \in C^{*} X$ and $\operatorname{supp}(f) \subset[a, b]$, then

$$
C^{*}|f|(x)=\int_{x}^{1} \frac{|f(t)|}{t} \mathrm{~d} t \geq \int_{a}^{b} \frac{|f(t)|}{t} \mathrm{~d} t \chi_{[0, a]}(x)=\|f\|_{L^{1}(1 / t)[0,1]} \chi_{[0, a]}(x) .
$$

Moreover,

$$
C^{*}|f|(x)=\int_{x}^{1} \frac{|f(t)|}{t} \mathrm{~d} t \leq \int_{a}^{b} \frac{|f(t)|}{t} \mathrm{~d} t \chi_{[0, b]}(x)=\|f\|_{L^{1}(1 / t)[0,1]} \chi_{[0, b]}(x) .
$$

Putting together the above inequalities we obtain 4.3.

The last part of this lemma is clear since

$$
\left\{f \in L^{1}(1 / t): \operatorname{supp}(f) \subset[a, b]\right\}=\left\{f \in L^{1}: \operatorname{supp}(f) \subset[a, b]\right\} \simeq L^{1}[0,1]
$$

whenever $0<a<b<1$ (because $\frac{1}{b}\left\|f \chi_{[a, b]}\right\|_{L^{1}[0,1]} \leq\left\|f \chi_{[a, b]}\right\|_{L^{1}(1 / t)[0,1]} \leq$ $\frac{1}{a}\left\|f \chi_{[a, b]}\right\|_{L^{1}[0,1]}$ for $\left.f \in L^{1}(1 / t)[0,1]\right)$ and it is enough to take the projection $P: f \mapsto f \chi_{[a, b]}$.

If additionally the Cesàro or the Copson operator is bounded on $X$, then we can deduce a little stronger versions of Lemma 4.1 and Lemma 4.2. respectively. More precisely, if $C$ is bounded on $X$, then Theorem $\mathrm{D}$ shows 
that $\operatorname{supp}(C X)=I$ and so the conclusion of Lemma 4.1 holds true for all $0<a<b<m(I)$. Of course, due to Corollary 3.2, an analogous remark is valid for every nontrivial $C^{*} X$.

It is clear that every nontrivial Cesàro or Copson function space contains a complemented copy of $\ell^{1}$ (simply because $L^{1}[0,1]$ does). Moreover, James's distortion theorem for $\ell^{1}$ states that a Banach space $X$ contains an isomorphic copy of $\ell^{1}$ if and only if it contains an almost isometric copy of $\ell^{1}$, that is, for every $0<\varepsilon<1$, there exists a sequence $\left(x_{n}\right) \subset X$ such that $(1-\varepsilon) \sum_{n=1}^{\infty}\left|\alpha_{n}\right| \leq\left\|\sum_{n=1}^{\infty} \alpha_{n} x_{n}\right\|_{X} \leq \sum_{n=1}^{\infty}\left|\alpha_{n}\right|$ for all $\alpha=\left(\alpha_{n}\right) \in \ell^{1}$. Therefore, as an immediate consequence of the complemented version of James's distortion theorem [32, Theorem 2] we obtain the following result.

Corollary 4.3. Let $T=C$ or $T=C^{*}$. If $X$ is a Banach function space on $I$ such that $T X \neq\{0\}$, then $T X$ contains a complemented almost isometric copy of $\ell^{1}$. In particular, $T X$ is not reflexive.

It turns out that we can even prove stronger versions of our Lemmas 4.1 and 4.2 and of [5, Proposition 2.2].

TheOREM 4.4. Let $X$ be a Banach space and assume that $X$ contains a complemented copy of a Banach space $Z$. Then there exists an equivalent norm on $X$ such that $X$ contains an isometric copy of $Z$. In particular, if $X$ is a Banach function space and $T=C$ or $T=C^{*}$, then:

(i) the space $T X$ can be renormed to contain an isometric copy of $L^{1}[0,1]$ whenever $T X \neq\{0\}$,

(ii) every nontrivial Tandori function space $\widetilde{X}$ can be renormed to contain an isometric copy of $L^{\infty}[0,1]$.

Proof. First, let $P: X \rightarrow X$ be a projection onto $Y_{1} \subset X$ and $T$ be an isomorphism from $Z$ onto $Y_{1}$. Consequently, we have the diagrams

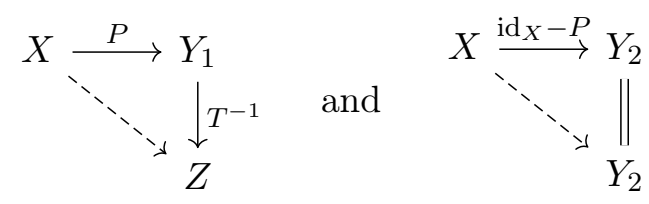

that is, $X \simeq Y_{1} \oplus Y_{2} \simeq Z \oplus Y_{2}$. We define a new norm $\|\cdot\| \|_{X}$ on $X$ by

$$
\|x\|_{X}:=\left\|T^{-1} P x\right\|_{Z}+\left\|\left(\operatorname{id}_{X}-P\right) x\right\|_{X} .
$$

This norm is equivalent to the original one: in fact,

$$
\begin{aligned}
\|x\|_{X} & \leq\left\|T^{-1}\right\|_{Y \rightarrow Z}\|P x\|_{Y}+(1+\|P\|)\|x\|_{X} \\
& \leq\left\|T^{-1}\right\|_{Y \rightarrow Z}\|P\|\|x\|_{X}+(1+\|P\|)\|x\|_{X} \\
& \leq\left(1+\|P\|+\left\|T^{-1}\right\|_{Y \rightarrow Z}\|P\|\right)\|x\|_{X},
\end{aligned}
$$


and

$$
\begin{aligned}
\|x\|_{X} & =\left\|P x+\left(\operatorname{id}_{X}-P\right) x\right\|_{X} \\
& \leq\|P x\|_{Y}+\left\|\left(\operatorname{id}_{X}-P\right) x\right\|_{X} \\
& \lesssim\left\|T^{-1} P x\right\|_{Z}+\left\|\left(\operatorname{id}_{X}-P\right) x\right\|_{X}=\|x\|_{X} .
\end{aligned}
$$

Moreover, if $y \in Y$ then

$$
\|y\|_{X}=\left\|T^{-1} P y\right\|_{Z}+\left\|\left(\operatorname{id}_{X}-P\right) y\right\|_{X}=\left\|T^{-1} P y\right\|_{Z}=\left\|T^{-1} y\right\|_{Z},
$$

which means that $T: Z \rightarrow\left(Y,\|\cdot \cdot\|_{X}\right)$ is an isometry.

(i) is clear due to Lemmas 4.1 and 4.2 and the first part of the proof.

(ii) Let us start with a simple observation. If $X$ is a Banach function space on $I$, then nontriviality of $\widetilde{X}$ is equivalent to $\chi_{[0, \lambda]} \in X$ for some $0<\lambda<m(I)$. Indeed, if $\widetilde{X} \neq\{0\}$, we can find $f \in \widetilde{X}$ such that $|f(x)|>0$ for $x \in A \subset I$ and $m(A)>0$. Setting $B_{n}:=\{x \in A:|f(x)|>1 / n\}$, where $n \in \mathbb{N}$, we see that there exists $n_{0} \in \mathbb{N}$ with $m\left(B_{n_{0}}\right)>0$. Therefore,

$$
\frac{1}{n_{0}} \chi_{\left[0, m\left(B_{n_{0}}\right)\right]} \leq \widetilde{f \chi_{B_{n_{0}}}} \leq \tilde{f} \in X,
$$

that is, $\chi_{[0, \lambda]} \in X$ for $\lambda=m\left(B_{n_{0}}\right)$. The converse implication is clear.

Now, since $\widetilde{X} \neq\{0\}$, it follows that $(0, \lambda) \subset \operatorname{supp}(\widetilde{X})$ for some $0<\lambda$ $<m(I)$. Using the same argument as in [5, Proposition 2.2] but for $0<a<$ $b<\lambda$ we can prove that $\widetilde{X}$ contains a complemented copy of $L^{\infty}[0,1]$. To finish the proof it is enough to apply the first part once again.

Note that if $X$ is a Banach function space on $I$ with $C X \neq\{0\}$, then exactly as in Theorem 4.4, we get the diagram

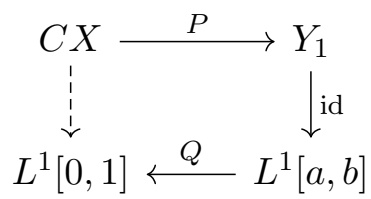

where $P:\left.f \mapsto f\right|_{[a, b]}$ for $0<a<b<m(I)$ is a bounded projection, $Y_{1}=$ $L^{1}[a, b]:=\left\{f \in L^{1}(I): \operatorname{supp}(f) \subset[a, b]\right\}, Q$ is a linear isometry between $L^{1}[a, b]$ and $L^{1}[0,1]$ and

$$
\|f\|_{C X}=\left\|f \chi_{[a, b]}\right\|_{L^{1}}+\left\|f \chi_{(0, a) \cup(b, m(I))}\right\|_{C X}
$$

for $f \in C X$. Now, if we take $X=L^{p}$ for $1 \leq p<\infty$ if $I=[0,1]$ and $1<p<\infty$ if $I=[0, \infty)$, then we obtain Astashkin-Maligranda's result [10, Lemma 4] concerning analogous renormings of classical Cesàro function spaces $\operatorname{Ces}_{p}$ (but the proof of Theorem 4.4 works in general and is completely different from that in [10, Lemma 4]).

Recall that a Banach function space $X$ contains an order asymptotically isometric copy of $\ell^{1}$ whenever there is a sequence $\left(f_{n}\right) \subset X$ with pairwise 
disjoint supports and a sequence $\left(\varepsilon_{n}\right) \subset(0,1)$ such that $\varepsilon_{n} \rightarrow 0$ and

$$
\sum_{n=1}^{\infty}\left(1-\varepsilon_{n}\right)\left|\alpha_{n}\right| \leq\left\|\sum_{n=1}^{\infty} \alpha_{n} f_{n}\right\|_{X} \leq \sum_{n=1}^{\infty}\left|\alpha_{n}\right|
$$

for each $\alpha=\left(\alpha_{n}\right) \in \ell^{1}$. This notion was introduced by Dowling-Lennard [29, Definition 1.1] and used to show that every nonreflexive subspace of $L^{1}[0,1]$ fails the fixed point property.

The notion of asymptotically isometric copy of $\ell^{1}$ is closely related to that of almost isometric copy of $\ell^{1}$ and consequently to James's distortion theorem (see [28, Question] and [44, p. 270]). However, Dowling-JohnsonLennard-Turett [28] gave an example of a renorming of $\ell^{1}$ which contains no asymptotically isometric copy of $\ell^{1}$. We will further extend the class of spaces which contain an asymptotically isometric copy of $\ell^{1}$ by showing that nontrivial Cesàro and Copson function spaces always contain such a copy. Note that for $\operatorname{Ces}_{p}$ this has been proved in [6. Theorems 1 and 2]. It would seem natural to first look for a generalization of this result to symmetric spaces (or even Orlicz spaces). Surprisingly, it turns out that symmetry of $X$ is not important in our proof.

Before giving the proof, for $X$ being a Banach function space on $I$ define a function $F_{X}$ as follows:

$$
F_{X}:=F_{X[0,1]}: I \ni \lambda \mapsto\left\|\frac{1}{x} \chi_{(\lambda, 1]}(x)\right\|_{X} \in[0, \infty]
$$

if $I=[0,1]$, and

$$
F_{X}:=F_{X[0, \infty)}: I \ni \lambda \mapsto\left\|\frac{1}{x} \chi_{(\lambda, \infty)}(x)\right\|_{X} \in[0, \infty]
$$

if $I=[0, \infty)$.

TheOREM 4.5. Let $X$ be a Banach function space on $I$ such that the Cesàro function space $C X$ is nontrivial. Then $C X$ contains an order asymptotically isometric copy of $\ell^{1}$.

Proof. $1^{\circ}$ Suppose $I=[0,1]$. Since $C X \neq\{0\}$, we have $\chi_{\left[\lambda_{0}, 1\right]} \in X$ for some $0<\lambda_{0}<1$ by Theorem D. For each $\lambda_{0}<a<1$ set

$$
\Omega_{a}:=\left\{\lambda \in\left(\lambda_{0}, 1\right): F_{X}(\lambda)=F_{X}(a)\right\} .
$$

Of course, $\operatorname{card}\left(\Omega_{a}\right) \geq 1$. Let us now consider the following two cases.

(a) Assume that $\operatorname{card}\left(\Omega_{a}\right)=1$ for every $a \in\left(\lambda_{0}, 1\right)$. Obviously, $F_{X}$ is nonincreasing in the interval $\left[\lambda_{0}, 1\right]$, so it has at most countably many points of discontinuity there. Let $\lambda_{0}<a_{0}<1$ be a point of continuity of $F_{X}$. Take a sequence $\left(a_{n}\right) \subset\left(\lambda_{0}, a_{0}\right)$ such that $a_{n} \uparrow a_{0}$ as $n \rightarrow \infty$ and put

$$
g_{n}:=\frac{\chi_{\left(a_{n}, a_{n+1}\right)}}{\left\|\chi_{\left(a_{n}, a_{n+1}\right)}\right\|_{C X}} .
$$


Then $\operatorname{supp}\left(g_{n}\right)=\left(a_{n}, a_{n+1}\right) \subset\left(a_{n}, a_{0}\right)$ and $\operatorname{supp}\left(g_{n}\right) \cap \operatorname{supp}\left(g_{m}\right)=\emptyset$ for $m, n \in \mathbb{N}$ distinct. Using the right-hand estimate of 4.1) we have

$$
\begin{aligned}
\left\|\chi_{\left(a_{n}, a_{n+1}\right)}\right\|_{C X} & \leq\left\|\frac{1}{x} \chi_{\left(a_{n}, 1\right]}(x)\right\|_{X}\left\|\chi_{\left(a_{n}, a_{n+1}\right)}\right\|_{L^{1}} \\
& =\left\|\frac{1}{x} \chi_{\left(a_{n}, 1\right]}(x)\right\|_{X}\left(a_{n+1}-a_{n}\right)=F_{X}\left(a_{n}\right)\left(a_{n+1}-a_{n}\right) .
\end{aligned}
$$

Furthermore, using the left inequality of (4.1) and the above estimate, since the functions $g_{n}$ have pairwise disjoint supports, we obtain

$$
\begin{aligned}
\left\|\sum_{n=1}^{\infty} \alpha_{n} g_{n}\right\|_{C X} & \geq\left\|\frac{1}{x} \chi_{\left[a_{0}, 1\right]}(x)\right\|\left\|_{X}\right\| \sum_{n=1}^{\infty} \alpha_{n} g_{n} \|_{L^{1}} \\
& =F_{X}\left(a_{0}\right)\left\|\sum_{n=1}^{\infty} \alpha_{n} g_{n}\right\|_{L^{1}}=F_{X}\left(a_{0}\right) \sum_{n=1}^{\infty} \frac{\left|\alpha_{n}\right|\left\|\chi_{\left(a_{n}, a_{n+1}\right)}\right\|_{L^{1}}}{\left\|\chi_{\left(a_{n}, a_{n+1}\right)}\right\|_{C X}} \\
& \geq F_{X}\left(a_{0}\right) \sum_{n=1}^{\infty} \frac{\left|\alpha_{n}\right|\left(a_{n+1}-a_{n}\right)}{F_{X}\left(a_{n}\right)\left(a_{n+1}-a_{n}\right)}=\sum_{n=1}^{\infty} \frac{F_{X}\left(a_{0}\right)}{F_{X}\left(a_{n}\right)}\left|\alpha_{n}\right|
\end{aligned}
$$

for each $\alpha=\left(\alpha_{n}\right) \in \ell^{1}$. Denote

$$
\theta_{n}:=\frac{F_{X}\left(a_{0}\right)}{F_{X}\left(a_{n}\right)} .
$$

Since $\operatorname{card}\left(\Omega_{a_{0}}\right)=1$, it follows that

$$
F_{X}\left(a_{n}\right)=\left\|\frac{1}{x} \chi_{\left(a_{n}, 1\right]}(x)\right\|_{X}>\left\|\frac{1}{x} \chi_{\left(a_{0}, 1\right]}(x)\right\|_{X}=F_{X}\left(a_{0}\right) .
$$

Consequently, $\left(\theta_{n}\right) \subset(0,1)$ and, thanks to continuity of $F_{X}$ at $a_{0}$, we have $\theta_{n} \rightarrow 1$ as $n \rightarrow \infty$. Finally, put

$$
\varepsilon_{n}:=1-\theta_{n} .
$$

Then $\left(\varepsilon_{n}\right) \subset(0,1), \varepsilon_{n} \rightarrow 0$ as $n \rightarrow \infty$ and

$$
\left\|\sum_{n=1}^{\infty} \alpha_{n} g_{n}\right\|_{C X} \geq \sum_{n=1}^{\infty}\left(1-\epsilon_{n}\right)\left|\alpha_{n}\right| .
$$

Note also that $\left\|g_{n}\right\|_{C X}=1$, so

$$
\left\|\sum_{n=1}^{\infty} \alpha_{n} g_{n}\right\|_{C X} \leq \sum_{n=1}^{\infty}\left|\alpha_{n}\right|\left\|g_{n}\right\|_{C X}=\sum_{n=1}^{\infty}\left|\alpha_{n}\right| .
$$

Combining 4.10 and 4.11, we finish the proof in this case.

(b) Assume that there is $a \in\left(\lambda_{0}, 1\right)$ with $\operatorname{card}\left(\Omega_{a}\right)>1$. Then there are $a_{1}, a_{2} \in\left(\lambda_{0}, 1\right)$ such that $a_{1}<a_{2}$ and $F_{X}\left(a_{1}\right)=F_{X}\left(a_{2}\right)$. Thus, for each $a_{3}$ with $a_{1}<a_{3}<a_{2}$, by the monotonicity of the norm, we have

$$
F_{X}\left(a_{1}\right) \geq F_{X}\left(a_{3}\right) \geq F_{X}\left(a_{2}\right),
$$


which means $F_{X}$ is constant on the interval $\left(a_{1}, a_{2}\right)$, i.e. $\left(a_{1}, a_{2}\right) \subset \Omega_{a}$. In the same way as in (a) we easily find that $C X$ contains an order isometric copy of $\ell^{1}$.

$2^{\circ}$ The proof when $I=[0, \infty)$ is the same as in the previous case. The only difference, of course, lies in the consideration of the function

$$
F_{X}:[0, \infty) \ni \lambda \mapsto\left\|\frac{1}{x} \chi_{(\lambda, \infty)}(x)\right\|_{X} \in(0, \infty] .
$$

It is clear that due to the similarities in Lemmas 4.1 and 4.2, a result analogous to Theorem 4.5 is expected also in the case of Copson function spaces. It will be convenient to start with the following natural modification of the function $F_{X}$ :

$$
G_{X}:=G_{X(I)}: I \ni \lambda \mapsto\left\|\chi_{[0, \lambda]}\right\|_{X(I)} \in(0, \infty],
$$

where $X$ is a Banach function space on $I$.

Theorem 4.6. Let $X$ be a Banach function space on I such that the Copson function space $C^{*} X$ is nontrivial. Then $C^{*} X$ contains an order asymptotically isometric copy of $\ell^{1}$.

Proof. With minor changes the proof is similar to that of Theorem 4.5 . Note, however, that the structure of the proof itself seems to be dual to the previous one. The details are provided for the convenience of the reader.

$1^{\circ}$ Suppose $I=[0,1]$. Since $C^{*} X \neq\{0\}$, there is $0<\lambda_{0}<1$ with $\chi_{\left[0, \lambda_{0}\right]} \in X$, by Lemma 3.1. For each $b \in\left(0, \lambda_{0}\right)$ set

$$
\Omega_{b}:=\left\{\lambda \in\left(0, \lambda_{0}\right): G_{X}(\lambda)=G_{X}(b)\right\} .
$$

Of course, $\operatorname{card}\left(\Omega_{b}\right) \geq 1$. Let us now consider the following two cases.

(a) Assume that card $\left(\Omega_{b}\right)=1$ for every $0<b<1$. Obviously, $G_{X}$ is nondecreasing on $\left[0, \lambda_{0}\right]$, so it has at most countably many points of discontinuity there. Let $b_{0} \in\left(0, \lambda_{0}\right)$ be a point of continuity of $G_{X}$. Take a sequence $\left(b_{n}\right) \subset\left(0, \lambda_{0}\right)$ such that $b_{n} \downarrow b_{0}$ as $n \rightarrow \infty$ and put

$$
h_{n}:=\frac{\chi_{\left(b_{n+1}, b_{n}\right)}}{\left\|\chi_{\left(b_{n+1}, b_{n}\right)}\right\|_{C^{*} X}} .
$$

Then $\operatorname{supp}\left(h_{n}\right)=\left(b_{n+1}, b_{n}\right) \subset\left(b_{0}, b_{n}\right)$ and $\operatorname{supp}\left(h_{n}\right) \cap \operatorname{supp}\left(h_{m}\right)=\emptyset$ for $m, n \in \mathbb{N}$ distinct. Using the right-hand inequality of 4.3 we have

$$
\begin{aligned}
\left\|\chi_{\left(b_{n+1}, b_{n}\right)}\right\|_{C^{*} X} & \leq\left\|\chi_{\left[0, b_{n}\right]}\right\| X\left\|\chi_{\left(b_{n+1}, b_{n}\right)}\right\|_{L^{1}(1 / t)} \\
& =G_{X}\left(b_{n}\right)\left\|\chi_{\left(b_{n+1}, b_{n}\right)}\right\|_{L^{1}(1 / t)} .
\end{aligned}
$$

Furthermore, using the left-hand inequality of 4.3 and the above estimate, 
since the functions $h_{n}$ have pairwise disjoint supports, we obtain

$$
\begin{aligned}
\left\|\sum_{n=1}^{\infty} \alpha_{n} h_{n}\right\|_{C^{*} X} & \geq\left\|\chi_{\left[0, b_{0}\right]}\right\|_{X}\left\|\sum_{n=1}^{\infty} \alpha_{n} h_{n}\right\|_{L^{1}(1 / t)} \\
& =G_{X}\left(b_{0}\right)\left\|\sum_{n=1}^{\infty} \alpha_{n} h_{n}\right\|_{L^{1}(1 / t)} \\
& =G_{X}\left(b_{0}\right) \sum_{n=1}^{\infty} \frac{\left|\alpha_{n}\right|\left\|\chi_{\left(b_{n+1}, b_{n}\right)}\right\|_{L^{1}(1 / t)}}{\left\|\chi_{\left(b_{n+1}, b_{n}\right)}\right\|_{C^{*} X}} \\
& \geq G_{X}\left(b_{0}\right) \sum_{n=1}^{\infty} \frac{\left|\alpha_{n}\right|\left\|\chi_{\left(b_{n+1}, b_{n}\right)}\right\|_{L^{1}(1 / t)}}{G_{X}\left(b_{n}\right)\left\|\chi_{\left(b_{n+1}, b_{n}\right)}\right\|_{L^{1}(1 / t)}}=\sum_{n=1}^{\infty} \frac{G_{X}\left(b_{0}\right)}{G_{X}\left(b_{n}\right)}\left|\alpha_{n}\right|
\end{aligned}
$$

for each $\alpha=\left(\alpha_{n}\right) \in \ell^{1}$. Denote

$$
\theta_{n}:=\frac{G_{X}\left(b_{0}\right)}{G_{X}\left(b_{n}\right)} .
$$

Since $\operatorname{card}\left(\Omega_{b_{0}}\right)=1$, it follows that $G_{X}\left(b_{n}\right)=\left\|\chi_{\left[0, b_{n}\right]}\right\|_{X}>\left\|\chi_{\left[0, b_{0}\right]}\right\|_{X}=$ $G_{X}\left(b_{0}\right)$. Consequently, $\left(\theta_{n}\right) \subset(0,1)$ and, thanks to continuity of $G_{X}$ at $b_{0}$, we have $\theta_{n} \rightarrow 1$ as $n \rightarrow \infty$. Finally, put $\varepsilon_{n}:=1-\theta_{n}$. Then $\left(\varepsilon_{n}\right) \subset(0,1)$, $\varepsilon_{n} \rightarrow 0$ as $n \rightarrow \infty$ and

$$
\left\|\sum_{n=1}^{\infty} \alpha_{n} h_{n}\right\|_{C^{*} X} \geq \sum_{n=1}^{\infty}\left(1-\epsilon_{n}\right)\left|\alpha_{n}\right| .
$$

Note also that $\left\|h_{n}\right\|_{C^{*} X}=1$, so

$$
\left\|\sum_{n=1}^{\infty} \alpha_{n} h_{n}\right\|_{C^{*} X} \leq \sum_{n=1}^{\infty}\left|\alpha_{n}\right|\left\|h_{n}\right\|_{C^{*} X}=\sum_{n=1}^{\infty}\left|\alpha_{n}\right| .
$$

Combining 4.15 and 4.16), we finish the proof in that case.

(b) Assume that there is $b \in\left(0, \lambda_{0}\right)$ with $\operatorname{card}\left(\Omega_{b}\right)>1$. Then there are $b_{1}, b_{2} \in(0,1)$ such that $b_{1}<b_{2}$ and $G_{X}\left(b_{1}\right)=G_{X}\left(b_{2}\right)$. Thus, for each $b_{3}$ with $b_{1}<b_{3}<b_{2}$,

$$
G_{X}\left(b_{1}\right) \leq G_{X}\left(b_{3}\right) \leq G_{X}\left(b_{2}\right),
$$

which means $G_{X}$ is constant on $\left(b_{1}, b_{2}\right)$, i.e., $\left(b_{1}, b_{2}\right) \subset \Omega_{b}$. As in case (a) we easily find that $C^{*} X$ contains an order isometric copy of $\ell^{1}$.

$2^{\circ}$ If $I=[0, \infty)$, we argue in the same way as in $1^{\circ}$.

In the context of Theorems 4.4 and 4.5 a natural question arises: maybe the Cesàro function space $\left(C X,\|\cdot\|_{C X}\right)$ always contains an isometric copy of $\ell^{1}$ or $L^{1}[0,1]$ ? In general, the answer is no. Indeed, if $X$ is rotund, then so is $C X$ [46, Lemma 2]. Therefore, for example, if $X=L^{p}$ for $1<p<\infty$, then $\operatorname{Ces}_{p}$ is rotund, so it cannot contain an isometric copy of $\ell^{1}$ or $L^{1}[0,1]$, 
because they are not rotund. However, $\left(C X,\|\cdot\|_{C X}\right)$ can always be renormed to contain an isometric copy of $L^{1}[0,1]$, by Theorem 4.4(i).

Theorem 4.5 also gives some information about generalized Tandori function spaces $X$ : they are quite similar to $L^{\infty}$.

Corollary 4.7. Let $X$ be a Banach function space on $I=[0, \infty)$ such that the space $X^{\prime}$ is order continuous and $X$ has the Fatou property (which is true, for example, if $X$ is reflexive). Assume also that the Copson operator $C^{*}: X \rightarrow X$ is bounded and the dilation operator $\sigma_{\tau}: X \rightarrow X$ is bounded for some $\tau>1$. Then the Tandori function space $\widetilde{X}$ contains an isomorphic copy of $L^{1}[0,1]$ and of $C[0,1]^{*}$.

Proof. Because $X^{\prime} \in(\mathrm{OC})$ we have $C\left(X^{\prime}\right) \in(\mathrm{OC})$. Note that since $X \in(\mathrm{FP}), C^{*}: X \rightarrow X$ if and only if $C: X^{\prime} \rightarrow X^{\prime}$ and $\sigma_{\tau}: X \rightarrow X$ if and only if $\sigma_{1 / \tau}: X^{\prime} \rightarrow X^{\prime}$ (see for example [49, Remark 1]). Consequently, applying (2.4) of Theorem A to $X^{\prime}$, we get

$$
\left(C\left(X^{\prime}\right)\right)^{*}=\left(C\left(X^{\prime}\right)\right)^{\prime}=\widetilde{X^{\prime \prime}}=\widetilde{X},
$$

with equivalent norms. The space $C\left(X^{\prime}\right)$ contains an order asymptotically isometric copy of $\ell^{1}$ via Theorem 4.5. By Dilworth-Girardi-Hagler's result [27. Theorem 2], the dual space $\left(C\left(X^{\prime}\right)\right)^{*}$ contains an isometric copy of $L^{1}[0,1]$ and an isometric copy of $C[0,1]^{*}$. Thus, by 44.17$), \widetilde{X}$ contains an isomorphic copy of $L^{1}[0,1]$ and of $C[0,1]^{*}$.

The similarity of $\widetilde{X}$ and $L^{\infty}$ becomes even clearer in the context of the following result.

Proposition 4.8. Let $X$ be a Banach function space on I such that the Tandori function space $\widetilde{X}$ is nontrivial. Then $\widetilde{X}$ contains an order isomorphically isometric copy of $\ell^{\infty}$.

Proof. Since $\widetilde{X} \neq\{0\}$, it follows that there exists $0<a \in I$ such that $\chi_{[0, a)} \in X$ [54, Theorem 1(c)]. Put

$$
f_{0}=\frac{\chi_{[0, a)}}{\left\|\chi_{[0, a)}\right\|_{X}} .
$$

Let $a_{n}=\left(1-\frac{1}{2^{n+1}}\right) a$ and $A_{n}=\left(a_{n}-\delta_{n}, a_{n}+\delta_{n}\right)$, where $\delta_{n}=\left(a_{n+1}-a_{n}\right) / 2$ and $n \in \mathbb{N}$. Denote

$$
B_{1}=[0, a / 2) \cup \bigcup_{n=1}^{\infty} A_{n} .
$$

Then $[0, a) \backslash B_{1}$ consists of infinitely many pairwise disjoint intervals, say $[0, a) \backslash B_{1}=\bigcup_{n=1}^{\infty} C_{n}^{(1)}$. Let

$$
B_{2}=C_{1}^{(1)} \cup C_{3}^{(1)} \cup C_{5}^{(1)} \cup \cdots=\bigcup_{n=1}^{\infty} C_{2 n-1}^{(1)} .
$$


Again, $[0, a) \backslash\left(B_{1} \cup B_{2}\right)$ consists of infinitely many pairwise disjoint intervals, say $[0, a) \backslash\left(B_{1} \cup B_{2}\right)=\bigcup_{n=1}^{\infty} C_{n}^{(2)}$. Next, let

$$
B_{3}=C_{1}^{(2)} \cup C_{3}^{(2)} \cup C_{5}^{(2)} \cup \cdots=\bigcup_{n=1}^{\infty} C_{2 n-1}^{(2)} .
$$

Proceeding analogously, we define a sequence $\left(B_{n}\right)_{n=1}^{\infty}$ of sets. Put $f_{n}:=$ $f_{0} \chi_{B_{n}}$. Note that

$$
0 \leq f_{n} \leq f_{0} \quad \text { and } \quad \operatorname{supp}\left(f_{n}\right) \cap \operatorname{supp}\left(f_{m}\right)=\emptyset \text { for } n \neq m .
$$

Moreover,

$$
\widetilde{f_{n}}=\widetilde{f_{0}}=f_{0}
$$

whence

$$
\left\|f_{n}\right\|_{\tilde{X}}=\left\|\widetilde{f}_{n}\right\|_{X}=\left\|\widetilde{f}_{0}\right\|_{X}=\left\|f_{0}\right\|_{X}=1 \text { and }\left\|f_{0}\right\|_{\widetilde{X}}=\left\|\widetilde{f}_{0}\right\|_{X}=\left\|f_{0}\right\|_{X}=1 .
$$

Applying [38, Theorem 1] we conclude that $\widetilde{X}$ contains an order isomorphically isometric copy of $\ell^{\infty}$.

The problem of describing the Cesàro-Orlicz function spaces containing an order isomorphically isometric copy of $\ell^{\infty}$ has been considered in [47. Although formally the case of $\mathrm{Ces}_{\infty}$ (which is not isomorphic to $\ell^{\infty}$ _ see [7, Theorem 7]) has been excluded there, the argument used in [47, Theorems 3 and 4 case (B2)] can be applied to get the result below. We will give, however, a direct and simple proof without referring to the Orlicz space structure.

Proposition 4.9. The space $\mathrm{Ces}_{\infty}$ contains an order isomorphically isometric copy of $\ell^{\infty}$.

Proof. For a start, let us recall that

$$
\left(\operatorname{Ces}_{\infty}\right)_{a}=\left\{f \in \operatorname{Ces}_{\infty}: \lim _{x \rightarrow 0^{+}, \infty} \frac{1}{x} \int_{0}^{x}|f(t)| \mathrm{d} t=0\right\}
$$

(see [48, Remark 19]). Put $f_{0}:=\chi_{[0,1]}$. Then

$$
\left\|f_{0}\right\|_{\operatorname{Ces}_{\infty}}=1 \text { and } \operatorname{dist}\left(f_{0},\left(\operatorname{Ces}_{\infty}\right)_{a}\right)=1 .
$$

Indeed, it follows from 4.18 that

$$
\begin{aligned}
\operatorname{dist}\left(f_{0},\left(\operatorname{Ces}_{\infty}\right)_{a}\right) & :=\inf _{h \in\left(\operatorname{Ces}_{\infty}\right)_{a}}\left\|f_{0}-h\right\|_{\operatorname{Ces}_{\infty}}=\inf _{h \in\left(\operatorname{Ces}_{\infty}\right)_{a}} \sup _{0 \leq t \in I} C\left|f_{0}-h\right|(t) \\
& \geq \inf _{h \in\left(\operatorname{Ces}_{\infty}\right)_{a}} \sup _{0 \leq t \in I}\left(C\left|f_{0}\right|(t)-C|h|(t)\right) \\
& \geq \inf _{h \in\left(\operatorname{Ces}_{\infty}\right)_{a}} \lim _{t \rightarrow 0^{+}}\left(C\left|f_{0}\right|(t)-C|h|(t)\right)=\lim _{t \rightarrow 0^{+}} C\left|f_{0}\right|(t)=1 .
\end{aligned}
$$

Now, we can use [38, Theorem 2] once again to finish the proof. 
5. Differences in the Cesàro construction on $[0,1]$ and $[0, \infty)$. We begin with a short discussion. Recall that following the standard definitions we should define a truncation of a Banach function space $X$ on $I$ to a set $A \subset I$ as $\left.X\right|_{A}=\{f \in X: \operatorname{supp}(f) \subset A\}$, where $\operatorname{supp}(f):=\{x \in I$ : $f(x) \neq 0\}$. However, when applying the Cesàro construction to the truncated space the situation is more delicate. If we wished to follow the above definition, we should define $C\left(\left.X[0, \infty)\right|_{[0,1]}\right)$ to be the space of all functions $f \in L^{0}[0, \infty)$ (because $\left.X[0, \infty)\right|_{[0,1]}$ contains functions from $\left.L^{0}[0, \infty)\right)$ such that $\left.C|f| \in X[0, \infty)\right|_{[0,1]}$, i.e., $f \in X[0, \infty)$ and $\operatorname{supp}(C|f|) \subset[0,1]$. But the last condition is never satisfied for $0 \neq f \in L^{0}[0, \infty)$, so this is meaningless.

That is why we will proceed in the following way. For a Banach function space $X$ on $[0, \infty)$ and a subset $A \subset[0, \infty)$ we define the truncation of the space $X[0, \infty)$ to a set $A$ as

$$
\left.X[0, \infty)\right|_{A}:=\left\{f \in L^{0}[0,1]: f \chi_{A} \in X[0, \infty)\right\},
$$

with the norm $\|f\|_{\left.X[0, \infty)\right|_{A}}=\left\|f \chi_{A}\right\|_{X[0, \infty)}$ (that is, we look at an element $f$ as a function defined on $[0,1]$, but compute its norm as if it was still defined on $[0, \infty)$ and equal to zero on $[0, \infty) \backslash A)$ if $A \subset[0,1]$, and

$$
\left.X[0, \infty)\right|_{A}:=\{f \in X[0, \infty): \operatorname{supp}(f) \subset A\},
$$

with the norm $\|f\|_{\left.X[0, \infty)\right|_{A}}=\|f\|_{X[0, \infty)}$, if $A \not \subset[0,1]$. Moreover, if $X$ is a Banach function space on $[0,1]$ and $A \subset[0,1]$, then we can use the definition given at the beginning, that is, the truncation of the space $X[0,1]$ to the set $A$ as

$$
\left.X[0,1]\right|_{A}:=\{f \in X[0,1]: \operatorname{supp}(f) \subset A\},
$$

with the norm $\|f\|_{\left.X[0,1]\right|_{A}}=\|f\|_{X[0,1]}$. Note that if we set

$$
\mathfrak{X}[0,1]:=\left.X[0, \infty)\right|_{[0,1]}=\left\{f \in L^{0}[0,1]: f \chi_{[0,1]} \in X[0, \infty)\right\},
$$

with the norm $\|f\|_{\mathfrak{X}}=\left\|f \chi_{[0,1]}\right\|_{X[0, \infty)}$, then the functor $X \mapsto \mathfrak{X}$ associates to the space $X$ defined on $I=[0, \infty)$ its "natural" counterpart defined on $I=[0,1]$. Observe also that if $A \subset[0,1]$, then the space $\left.X[0, \infty)\right|_{A}$ is actually isometrically isomorphic to the space $Y:=\{f \in X[0, \infty): \operatorname{supp}(f) \subset A\}$ with the norm $\|f\|_{Y}=\|f\|_{X[0, \infty)}$ via the mapping $J: f \mapsto f \chi_{A}$. Moreover, it is clear that $\left.X[0, \infty)\right|_{[0,1]}$ is a symmetric space whenever $X[0, \infty)$ is.

Clearly, $\left.L^{p}[0, \infty)\right|_{[0,1]} \equiv L^{p}[0,1]$. In contrast, for Cesàro function spaces the situation is quite different. In fact, if $f \in \operatorname{Ces}_{p}[0, \infty)$ and $\operatorname{supp}(f) \subset[0,1]$, then

$$
\|f\|_{\operatorname{Ces}_{p}[0, \infty)}^{p}=\|f\|_{\operatorname{Ces}_{p}[0,1]}^{p}+\frac{1}{p-1}\|f\|_{L^{1}[0,1]}^{p},
$$

i.e., $\left.\operatorname{Ces}_{p}[0, \infty)\right|_{[0,1]}=\operatorname{Ces}_{p}[0,1] \cap L^{1}[0,1]$ for $1<p<\infty$ [7, Remark 5]. In the next lemma we will give an analogue of $(5.5)$ for abstract Cesàro function 
spaces $C X$. However, notice that according to the definitions (5.1) and (5.2), we have

$$
\begin{aligned}
\left.C X[0, \infty)\right|_{[0,1]} & =\left.(C X[0, \infty))\right|_{[0,1]} \\
& :=\left\{f \in L^{0}[0,1]: C\left(|f| \chi_{[0,1]}\right) \in X[0, \infty)\right\},
\end{aligned}
$$

with the norm $\|f\|_{\left.C X[0, \infty)\right|_{[0,1]}}=\left\|C\left(|f| \chi_{[0,1]}\right)\right\|_{X[0, \infty)}$, and

$$
\begin{aligned}
C\left(\left.X[0, \infty)\right|_{[0,1]}\right): & =C(\mathfrak{X}[0,1]) \\
& =\left\{f \in L^{0}[0,1]:(C|f|) \chi_{(0,1]} \in X[0, \infty)\right\},
\end{aligned}
$$

with the norm $\|f\|_{C\left(\left.X[0, \infty)\right|_{[0,1]}\right)}=\left\|(C|f|) \chi_{(0,1]}\right\|_{X[0, \infty)}$ (cf. with the definition of the Cesàro operator $C$ and the abstract Cesàro space $C X$ ). Roughly speaking, if $X$ is a Banach function space on $[0, \infty)$, then we always have two ways (in general, inequivalent) to obtain the Cesàro function space on $[0,1]$ : first applying the functor $X \mapsto \mathfrak{X}$ and then the Cesàro construction, or the other way round. All this also means that the Cesàro construction $X \mapsto C X$ is significantly different for $I=[0,1]$ and for $I=[0, \infty)$.

The equality (5.9) from Lemma 5.1 below is an abstract version of the equality (5.5) from [7] and means that the functor $X \mapsto \mathfrak{X}$ does not commute in general with the Cesàro construction $X \mapsto C X$. This result also explains, in some sense, a rather suprising difference in the description of the Köthe duality of Cesàro function spaces $C X$ on $I=[0,1]$ and on $I=[0, \infty)$ : cf. [54, Theorems 3,5 and 6] or Theorem A.

Lemma 5.1. Let $X$ be a Banach function space on I such that either the Cesàro operator $C$ is bounded on $X$ or $X$ is a symmetric space on $[0,1]$ or $X$ is a symmetric space on $[0, \infty)$ with $C X[0, \infty) \neq\{0\}$. Then the embedding

$$
\left.\left.C X(I)\right|_{[0, \lambda]} \hookrightarrow L^{1}(I)\right|_{[0, \lambda]}
$$

holds for $0<\lambda<m(I)$, but in general not for $\lambda=1$ if $I=[0,1]$, and not for $\lambda=\infty$ if $I=[0, \infty)$. Moreover,

$$
\begin{aligned}
& \left.C X[0, \infty)\right|_{[0,1]}=C\left(\left.X[0, \infty)\right|_{[0,1]}\right) \cap L^{1}[0,1], \\
& \left.C X[0, \infty)\right|_{[0, \lambda]}=\left.\left(C\left(\left.X[0, \infty)\right|_{[0,1]}\right)\right)\right|_{[0, \lambda]} \quad \text { for } 0<\lambda<1 .
\end{aligned}
$$

Finally, if $X$ is a symmetric space on $[0, \infty)$ with $q(X)<\infty$, then $C\left(\left.X[0, \infty)\right|_{[0,1]}\right) \neq\left. C X[0, \infty)\right|_{[0,1]}$ and $C\left(\left.X[0, \infty)\right|_{[0,1]}\right)$ is never a subspace of $\left.C X[0, \infty)\right|_{[0,1]}$.

Proof of (5.8). Take $f \in C X$ with $\operatorname{supp}(f) \subset[0, \lambda]$, where $0<\lambda<m(I)$. In this case we must ensure that $\left(f_{\lambda}: I \ni x \mapsto \frac{1}{x} \chi_{(\lambda, m(I))}(x)\right) \in X$. But this immediately follows from Theorem D and our assumptions. Keeping this in 
mind we have

$$
\begin{aligned}
\|f\|_{C X} & :=\left\|\frac{1}{x} \int_{0}^{x}|f(t)| \mathrm{d} t\right\|_{X} \\
& =\left\|\frac{1}{x} \int_{0}^{x}|f(t)| \mathrm{d} t \chi_{(0, \lambda]}(x)+\frac{1}{x} \int_{0}^{\lambda}|f(t)| \mathrm{d} t \chi_{(\lambda, m(I))}(x)\right\|_{X} \\
& \geq\left\|\frac{1}{x} \int_{0}^{\lambda}|f(t)| \mathrm{d} t \chi_{(\lambda, m(I))}(x)\right\|_{X}=\left\|\frac{1}{x} \chi_{(\lambda, m(I))}(x)\right\|_{X}\|f\|_{\left.L^{1}\right|_{[0, \lambda]}} .
\end{aligned}
$$

Therefore, $\left.\left.C X\right|_{[0, \lambda]} \hookrightarrow L^{1}\right|_{[0, \lambda]}$. Counterexamples to embeddings $C X \hookrightarrow L^{1}$ when either $\lambda=1$ or $\lambda=\infty$ can be found in [7, Theorem 1(d)]. Note, however, that for example $\operatorname{Ces}_{\infty}[0,1] \hookrightarrow L^{1}[0,1]$ [7. Theorem $\left.1(\mathrm{~d})\right]$.

Proof of (5.9). Take $f \in C\left(\left.X[0, \infty)\right|_{[0,1]}\right) \cap L^{1}[0,1]$. To prove the desired equality we will need to know that $\left(f_{\lambda=1}:[0, \infty) \ni x \mapsto \frac{1}{x} \chi_{(1, \infty)}(x)\right) \in$ $X[0, \infty)$. Again, our assumptions together with Theorem $\mathrm{D}$ ensure that this is indeed the case. Since

$$
\begin{aligned}
\|f\|_{C X[0, \infty)} \leq & \left\|\frac{1}{x} \int_{0}^{x}|f(t)| \mathrm{d} t \chi_{(0,1]}(x)\right\|_{X[0, \infty)} \\
& +\left\|\frac{1}{x} \int_{0}^{1}|f(t)| \mathrm{d} t \chi_{(1, \infty)}(x)\right\|_{X[0, \infty)} \\
= & \|f\|_{C\left(\left.X[0, \infty)\right|_{[0,1]}\right)}+\left\|\frac{1}{x} \chi_{(1, \infty)}(x)\right\|_{X[0, \infty)}\|f\|_{L^{1}[0,1]}
\end{aligned}
$$

it follows that

$$
\left.C\left(\left.X[0, \infty)\right|_{[0,1]}\right) \cap L^{1}[0,1] \hookrightarrow C X[0, \infty)\right|_{[0,1]} .
$$

Next, we will show the reverse embedding. Take $f \in C X[0, \infty)$ with $\operatorname{supp}(f) \subset[0,1]$. Again, as above, we have $\frac{1}{x} \chi_{(1, \infty)}(x) \in X$, whence

$$
\begin{aligned}
\|f\|_{C X[0, \infty)} & \geq \max \left\{\left\|(C|f|) \chi_{(0,1)}\right\|_{X[0, \infty)},\left\|\frac{1}{x} \int_{0}^{1}|f(t)| \mathrm{d} t \chi_{(1, \infty)}(x)\right\|_{X[0, \infty)}\right\} \\
& =\max \left\{\|f\|_{C\left(\left.X[0, \infty)\right|_{[0,1]}\right)},\left\|\frac{1}{x} \chi_{(1, \infty)}(x)\right\|_{X[0, \infty)}\|f\|_{L^{1}[0,1]}\right\} .
\end{aligned}
$$

In consequence,

$$
\left.C X[0, \infty)\right|_{[0,1]} \hookrightarrow C\left(\left.X[0, \infty)\right|_{[0,1]}\right) \cap L^{1}[0,1],
$$

which proves 5.9 . 
Proof of (5.10). Of course, if $X$ and $Y$ are Banach function spaces on $I$, then

$$
\left.\left.\left.(X \cap Y)\right|_{A} \equiv X\right|_{A} \cap Y\right|_{A} \quad \text { for every } A \subset I,
$$

and because $\|f\|_{\left.\left(\left.X\right|_{A}\right)\right|_{B}}=\left\|f \chi_{B}\right\|_{\left.X\right|_{A}}=\left\|f \chi_{B} \chi_{A}\right\|_{X}=\left\|f \chi_{A \cap B}\right\|_{X}=\|f\|_{\left.X\right|_{A \cap B}}$, we have

$$
\left.\left.\left(\left.X\right|_{A}\right)\right|_{B} \equiv X\right|_{A \cap B} \quad \text { for all } A, B \subset I .
$$

Combining the above equalities with the embedding (5.8) we get

$$
\begin{aligned}
\left.C X[0, \infty)\right|_{[0, \lambda]} & \left.\equiv\left(\left.C X[0, \infty)\right|_{[0,1]}\right)\right|_{[0, \lambda]}=\left.\left(C\left(\left.X[0, \infty)\right|_{[0,1]}\right) \cap L^{1}[0,1]\right)\right|_{[0, \lambda]} \\
& =\left.\left(C\left(\left.X[0, \infty)\right|_{[0,1]}\right)\right)\right|_{[0, \lambda]} .
\end{aligned}
$$

This gives 5.10).

Finally, suppose that $q(X)<\infty$. Then as in [7, Theorem 1(d)] we can show that the function

$$
f(x)=\frac{1}{1-x} \quad \text { for } 0 \leq x<1
$$

belongs to the space $C\left(\left.X[0, \infty)\right|_{[0,1]}\right)$. In fact, by $(2.6)$ Theorem $\mathrm{B}$, we have $L^{1} \cap L^{q}[0, \infty) \stackrel{A}{\hookrightarrow} X[0, \infty)$ for $q(X)<q<\infty$. Therefore, using 5.11 we have

$$
L^{q}[0,1]=L^{1} \cap L^{q}[0,1]=\left.\left.\left(L^{1} \cap L^{q}[0, \infty)\right)\right|_{[0,1]} \stackrel{A}{\hookrightarrow} X[0, \infty)\right|_{[0,1]} .
$$

Moreover, $\int_{0}^{1}\left(\frac{1}{x} \ln \left(\frac{1}{1-x}\right)\right)^{q} \mathrm{~d} x<\infty$ [7, Theorem 1(d), p. 334], whence

$$
\begin{aligned}
\|f\|_{C\left(\left.X[0, \infty)\right|_{[0,1]}\right)}^{q} & =\|C|f|\|_{\left.X[0, \infty)\right|_{[0,1]}}^{q} \lesssim\|C|f|\|_{L^{q}[0,1]}^{q} \\
& =\int_{0}^{1}\left(\frac{1}{x} \int_{0}^{x} \frac{\mathrm{d} t}{1-t}\right)^{q} \mathrm{~d} x=\int_{0}^{1}\left(\frac{1}{x} \ln \left(\frac{1}{1-x}\right)\right)^{q} \mathrm{~d} x<\infty .
\end{aligned}
$$

Of course, $f \notin L^{1}[0,1]$ and this ends the proof of the lemma.

Also note that if $X=L^{\infty}[0, \infty)$, then $q(X)=\infty$ and $\left.L^{\infty}[0, \infty)\right|_{[0,1]} \equiv$ $L^{\infty}[0,1]$. Therefore, $C\left(\left.X[0, \infty)\right|_{[0,1]}\right)=\operatorname{Ces}_{\infty}[0,1]$ and, in view of $(5.9)$ and $\operatorname{Ces}_{\infty}[0,1] \hookrightarrow L^{1}[0,1][7$, Theorem $1(\mathrm{~d})]$, we have

$$
\left.C X[0, \infty)\right|_{[0,1]}=\left.\operatorname{Ces}_{\infty}[0, \infty)\right|_{[0,1]}=\operatorname{Ces}_{\infty}[0,1] \cap L^{1}[0,1]=\operatorname{Ces}_{\infty}[0,1] .
$$

This means that it can happen that $C\left(\left.X[0, \infty)\right|_{[0,1]}\right)=\left.C X[0, \infty)\right|_{[0,1]}$ and the assumption about the Boyd index in the last part of the above lemma cannot be omitted.

6. Transfer of properties between $X$ and $T X$. Inclusions and equalities between Cesàro spaces $C X$ and Copson spaces $C^{*} X$ are collected, for example, in [56, Theorem 1] (see also [7] and [13]). Recall that if $X$ is a 
Banach function space on $[0, \infty)$ such that both operators $C$ and $C^{*}$ are bounded on $X$, then

$$
C^{*} X \stackrel{A}{\hookrightarrow} C X \stackrel{B}{\hookrightarrow} C^{*} X,
$$

where $A=\|C\|_{X \rightarrow X}$ and $B=\left\|C^{*}\right\|_{X \rightarrow X}$, that is, $C X=C^{*} X$ [56, Theorem 1(iii)]. However, if $I=[0,1]$, then the situation is a bit more complicated. More precisely, if $X$ is a Banach function space on $[0,1]$ such that the Cesàro and Copson operators are bounded on $X$ and $L^{\infty}[0,1] \hookrightarrow X \hookrightarrow L^{1}[0,1]$ (for example, if $X$ is a symmetric space), then

$$
C X[0,1] \cap L^{1}[0,1]=C^{*} X[0,1]
$$

(see [56, Theorem 1(vi, vii)]). Therefore, at least when we consider Banach function spaces on $[0, \infty)$ such that both $C$ and $C^{*}$ are bounded on $X$, all results regarding the isomorphic structure of Cesàro function spaces "transfer" almost trivially to Copson function spaces and vice versa. However, it may not be the case if $I=[0,1]$. Nonetheless, we will prove

Corollary 6.1. Let $X$ be a symmetric space such that both operators $C$ and $C^{*}$ are bounded on $X$. Then the Copson space $C^{*} X$ is order continuous if and only if $X$ is order continuous.

Proof. First of all, since $X$ is a symmetric space and $C$ is bounded on $X$, the space $X$ is order continuous if and only if $C X$ is [48, Theorem 3]. Thus, according to 6.1 and the discussion preceding it, there is nothing to prove when $I=[0, \infty)$. Therefore, let $I=[0,1]$.

Suppose that $X \in(\mathrm{OC})$. It follows from [56, Lemma 1(a)] that $C X \in(\mathrm{OC})$, so $C X \cap L^{1} \in(\mathrm{OC})$. Using 6.2 we see immediately that $C^{*} X \in(\mathrm{OC})$.

To prove the reverse implication, assume that $X \notin(\mathrm{OC})$. Because $X$ is a symmetric space, we can find $f_{0} \in X$ such that $f_{0} \notin X_{a}$ but $f_{0} \in L^{1}[0,1]$. Without loss of generality, we can also assume that $f_{0}=f_{0}^{*}$ [17, Lemma 2.6]. From the boundedness of $C$ it follows that $C\left(f_{0}\right) \in X$. Moreover, $f_{0}$ is a nonincreasing function and $X_{a}$ is an order ideal of $X$ 114, Theorem 3.8, p. 16], so $C\left(f_{0}\right) \geq f_{0}$ and also $C\left(f_{0}\right) \notin X_{a}$. Therefore, $f_{0} \notin C\left(X_{a}\right)=(C X)_{a}$ [48, Theorem 16]. In summary, $f_{0} \in\left(C X \backslash(C X)_{a}\right) \cap L^{1}[0,1]$. But, in view of (6.2), this means that $f_{0} \in C^{*} X \backslash\left(C^{*} X\right)_{a}$, i.e., $C^{*} X \notin(\mathrm{OC})$.

It may happen that applying the construction $X \mapsto T X$, where $T=C$ or $T=C^{*}$, we lose some information about the original space $X$. We will give a rather general example of this kind.

The idea behind the next lemma is simple. Every Cesàro and Copson function space contain "in the middle" an isomorphic copy of $L^{1}[0,1]$ (cf. Lemmas 4.1 and 4.2 . Therefore, up to equivalence of norms, we can change the space $X$ "in the middle" (cf. (6.3)) and still get the Cesàro or Copson function space equal to the original one (cf. (6.4)). 
Lemma 6.2. Let $T=C$ or $T=C^{*}$. Define the Banach function space $Z=Z[0,1]$ as

$$
Z[0,1]:=\left.\left.\left.X\right|_{[0, a]} \oplus Y\right|_{[a, b]} \oplus X\right|_{[b, 1]} \quad \text { for } 0<a<b<1,
$$

where $X$ and $Y$ are Banach function spaces on $[0,1]$ such that $L^{\infty}[0,1] \hookrightarrow$ $Y \hookrightarrow X$. Then

$$
T Z[0,1]=T X[0,1] .
$$

Proof of $C Z \hookrightarrow C X$ and $C^{*} Z \hookrightarrow C^{*} X$. In the proof we need only the assumption that $Y \hookrightarrow X$. Indeed, then

$$
Z[0,1]=\left.\left.\left.\left.\left.\left.X\right|_{[0, a]} \oplus Y\right|_{[a, b]} \oplus X\right|_{[b, 1]} \hookrightarrow X\right|_{[0, a]} \oplus X\right|_{[a, b]} \oplus X\right|_{[b, 1]}=X[0,1] .
$$

Note that just by the definition, if $E$ and $F$ are Banach function spaces on $I$ and $E \hookrightarrow F$, then $T E \hookrightarrow T F$. Consequently, $T Z \hookrightarrow T X$.

Proof of $C X \hookrightarrow C Z$. First, observe that if $W$ is a Banach function space on $[0,1] \operatorname{such}$ that $\operatorname{supp}(C W)=[0,1]$, then

$$
\left.(C W[0,1])\right|_{[a, b]}=\left.L^{1}[0,1]\right|_{[a, b]}=L^{1}[a, b] \quad \text { for } 0<a<b<1,
$$

due to Lemma 4.1. Take $f \in C X$ and denote $f_{1}=f \chi_{[0, a)}, f_{2}=f \chi_{[a, b)}$ and $f_{3}=f \chi_{[b, 1]}$. We need to show that $f_{1}, f_{2}, f_{3} \in C Z$. Note that $\left(C\left|f_{1}\right|\right) \chi_{[0, a)}=$ $(C|f|) \chi_{[0, a)} \in X$. Moreover, $\left(C\left|f_{1}\right|\right) \chi_{[a, b)} \in L^{\infty}[0,1]$, so $\left(C\left|f_{1}\right|\right) \chi_{[a, b)} \in Y$, because $L^{\infty}[0,1] \hookrightarrow Y$. Next, observe that $\left(C\left|f_{1}\right|\right) \chi_{[b, 1)} \leq(C|f|) \chi_{[b, 1)} \in X$. Thus $C\left|f_{1}\right| \in Z$. Since $L^{\infty}[0,1] \hookrightarrow Y \hookrightarrow X$, so $\quad L^{\infty}[0,1] \hookrightarrow Z$ and $\operatorname{supp}(C X)=$ $\operatorname{supp}(C Z)=[0,1]$. In consequence, by equality $(6.5)$, we have $\left.f_{2} \in C X\right|_{[a, b]}=$ $\left.L^{1}[0,1]\right|_{[a, b]}=\left.C Z\right|_{[a, b]}$. Finally, $C\left|f_{3}\right|=C\left(|f| \chi_{[b, 1]}\right) \leq\left. C(|f|) \chi_{[b, 1]} \in X\right|_{[b, 1]}$ $=\left.Z\right|_{[b, 1]}$. Consequently, $f \in C Z$, but this means that $C X \hookrightarrow C Z$.

Proof of $C^{*} X \hookrightarrow C^{*} Z$. Let $f \in C^{*} X$ and set $f_{1}=f \chi_{[0, a)}, f_{2}=f \chi_{[a, b)}$ and $f_{3}=f \chi_{[b, 1]}$. Since $C^{*}\left|f_{1}\right| \leq C^{*}|f| \in X$, it follows that $\left(C^{*}\left|f_{1}\right|\right) \chi_{[0, a)} \in Z$. Moreover, $\left(C^{*}\left|f_{1}\right|\right) \chi_{[a, 1]} \equiv 0$, whence $f_{1} \in C Z$. Just as above we conclude that $\operatorname{supp}\left(C^{*} X\right)=\operatorname{supp}\left(C^{*} Z\right)=[0,1]$. Thus, by Lemma 4.2, $\left.f_{2} \in C^{*} X\right|_{[a, b]}=$ $L^{1}[a, b]=\left.C^{*} Z\right|_{[a, b]}$. Moreover, $C^{*}\left|f_{3}\right| \leq C^{*}|f| \in X$, so $\left(C^{*}\left|f_{3}\right|\right) \chi_{[0, a)} \in Z$ and $\left(C^{*}\left|f_{3}\right|\right) \chi_{[b, 1]} \in Z$. Finally, note that $C^{*}|f| \in X$ and the function $C^{*}|f|$ is nonincreasing, thus $\left(C^{*}\left|f_{3}\right|\right) \chi_{[a, b)}(x)=\int_{b}^{1} \frac{|f(t)|}{t} d t=C^{*}|f|(b)<\infty$ for each $x \in[a, b)$. Therefore, we find that $\left.\left.\left(C^{*}\left|f_{3}\right|\right) \chi_{[a, b)} \in L^{\infty}[0,1]\right|_{[a, b]} \hookrightarrow Y\right|_{[a, b]}$ and this ends the proof.

The above lemma can be viewed as a far-reaching generalization of 56, Example 1]. In particular, if $X$ is a symmetric space on $[0,1]$, then $L^{\infty}[0,1] \hookrightarrow$ $X \hookrightarrow L^{1}[0,1]$,

$$
T\left(\left.L^{1}[0, a] \oplus X\right|_{[a, b]} \oplus L^{1}[b, 1]\right) \simeq L^{1}[0,1]
$$

and

$$
T\left(\left.\left.X\right|_{[0, a]} \oplus L^{\infty}[a, b] \oplus X\right|_{[b, 1]}\right)=T X[0,1] .
$$


In connection with the above lemma, the following simple observation is worth noting.

Lemma 6.3. Let $T=C$ or $T=C^{*}$. Assume that $X$ and $Y$ are symmetric spaces on I such that

(i) $C$ is bounded on $X$ and $Y$ if $T=C$,

(ii) $C$ and $C^{*}$ are bounded on $X$ and $Y$, respectively, if $T=C^{*}$.

Then $X=Y$ if and only if $T X=T Y$.

Proof. If $X=Y$, then $X \hookrightarrow Y$ and $Y \hookrightarrow X$, so $T X \hookrightarrow T Y$ and $T Y \hookrightarrow T X$. Thus, $T X=T Y$.

Suppose that $T=C$. If $C X \hookrightarrow C Y$, then thanks to boundedness of the Cesàro operator and the symmetry of $X$, we have

$$
\|f\|_{Y}=\left\|f^{*}\right\|_{Y} \leq\left\|C\left(f^{*}\right)\right\|_{Y} \lesssim\left\|C\left(f^{*}\right)\right\|_{X} \leq\|C\|_{X \rightarrow X}\left\|f^{*}\right\|_{X} \lesssim\|f\|_{X},
$$

that is, $X \hookrightarrow Y$. Interchanging the roles of $X$ and $Y$ we can show the reverse embedding $Y \hookrightarrow X$.

Let $T=C^{*}$ and assume that $C^{*} X \hookrightarrow C^{*} Y$. If $I=[0, \infty)$, then $C^{*} X=C X$ and there is nothing to prove (see (6.1)). On the other hand, if $I=[0,1]$, then $C^{*} X=C X \cap L^{1}$ (see (6.2)). Since $X \hookrightarrow C^{*} X$, it follows that

$$
X \hookrightarrow C^{*} X \hookrightarrow C^{*} Y=C Y \cap L^{1} \hookrightarrow C Y .
$$

Moreover, $\|f\|_{Y}=\left\|f^{*}\right\|_{Y} \leq\left\|C\left(f^{*}\right)\right\|_{Y}$ and consequently

$$
\|f\|_{Y} \leq\left\|C\left(f^{*}\right)\right\|_{Y}=\left\|f^{*}\right\|_{C Y} \lesssim\left\|f^{*}\right\|_{X}=\|f\|_{X},
$$

that is, $X \hookrightarrow Y$.

If we try to reformulate Lemma 6.3 using isomorphism instead of "equalities", then this result is not longer true. For example, if $X=L^{\infty}$ and $Y=L^{\infty}(t)$, then $C Y \equiv L^{1}$ and $X \simeq Y$ but of course $L^{1}$ is not isomorphic to $\operatorname{Ces}_{\infty}$ (because $L^{1}$ is separable and $\operatorname{Ces}_{\infty}$ is not). On the other hand, if $X=L^{1}[0,1]$ and $Y=L^{\infty}(t)[0,1]$, then $C X \simeq L^{1}[0,1], C Y \equiv L^{1}[0,1]$ and $C X \simeq C Y$ but $X$ is not isomorphic to $Y$.

Generally, some isomorphic as well as isometric properties pass from $X$ to $C X$ (for example, order continuity [56], Fatou property [54, Theorem 1(d)] and rotundity [46]). However, there are properties, like reflexivity, which Cesàro function spaces never have (cf. Corollary 4.3). In other words, certain properties never transfer from $X$ to $C X$. Below we present another two properties of this kind.

Corollary 6.4. Let $T=C$ or $T=C^{*}$. Suppose that $X$ is an order continuous Banach function space on $I$ with $T X \neq\{0\}$. Then

(i) $T X$ is not isomorphic to a dual space,

(ii) $T X$ does not have the Radon-Nikodym property. 
Proof. The proof of (i) is the same as in [10, Theorem 3]. We give the details for completeness.

(i) Suppose for contradiction that $T X$ is isomorphic to a dual space, i.e. there exists a Banach space $Y$ with $\left(T X,\|\cdot\|_{T X}\right) \simeq Y^{*}$. By Theorem 4.4 we can find an equivalent norm, say $\|\cdot\|^{\prime}$, on $T X$ such that $\left(T X,\|\cdot\|^{\prime}\right)$ contains a closed subspace isometric to $L^{1}[0,1]$. Of course, $\left(T X,\|\cdot\|^{\prime}\right) \simeq Y^{*}$. It follows from the definition that if $X \in(\mathrm{OC})$ then $T X \in(\mathrm{OC})$ (cf. also [56, Lemma 1(a)]). Thus, our assumptions show that $\left(T X,\|\cdot\|^{\prime}\right) \in(\mathrm{OC})$. Now, we can apply the well-known fact that a Banach function space $X$ relative to a measure $\mu$ is separable if and only if it is order continuous and the measure $\mu$ is separable [14, Theorem 5.5] to conclude that $\left(T X,\|\cdot\|^{\prime}\right)$ is also separable. Applying Bessaga-Pełczyński's result [15], it follows that $\left(T X,\|\cdot\|^{\prime}\right)$ has the Krein-Milman property. Therefore, every closed bounded set in $\left(T X,\|\cdot\|^{\prime}\right)$ is the closed convex hull of its extreme points. On the other hand, the closed unit ball in $L^{1}[0,1]$ has no extreme points. This contradiction ends the proof.

(ii) This case follows from the Talagrand theorem [63, Corollary 5.4.21], which states that a separable Banach lattice is isomorphic to a dual Banach lattice if and only if it has the Radon-Nikodym property.

The above corollary has been proved for $\mathrm{Ces}_{p}$ in [10, Theorem 3] and in [41, Corollaries 5.1 and 5.5] (using duality arguments). Moreover, part (i) for $C X$, where $X$ is an order continuous symmetric space such that $C$ is bounded on $X$, is included in [5, Proposition 5.3]. Interestingly, it may happen that $C X$, for a nonseparable $X$, is isomorphic to a dual space. It was proved in [5, (2.9) and Theorem 5.1] that

$$
\left(\widetilde{\ell^{1}}\right)^{*}=\left(\widetilde{\ell^{1}}\right)^{\prime}=\operatorname{ces}_{\infty} \simeq \operatorname{Ces}_{\infty},
$$

which means that $\mathrm{Ces}_{\infty}$ is isomorphic to a dual space.

The question arises when a given property "transfers" also in the opposite direction, i.e. from $C X$ to $X$. However, as the next theorem will show, in the class of Banach function spaces the answer is basically always negative. Before we formulate this result we need the following definition.

Let $P$ be a property defined for Banach function spaces. We will say that the property $P$ is good for the Cesàro construction if

$1^{\circ} P$ is invariant under order isomorphisms (that is, if $\left(X,\|\cdot\|_{X}\right) \in(P)$ and $T$ : $\left(X,\|\cdot\|_{X}\right) \rightarrow\left(Y,\|\cdot\|_{Y}\right)$ is an order isomorphism, then also $\left.\left(Y,\|\cdot\|_{Y}\right) \in(P)\right)$, $2^{\circ}$ for any Banach function space $Z$ on $[0,1]$, if $Z \in(P)$ then $\left.Z\right|_{[a, b]} \in(P)$ for every $0<a<b<1$,

$3^{\circ}$ there exist nontrivial Banach function spaces $X$ and $Y$ on $[0,1]$ such that

(G1) $C X \neq\{0\}$ and $C X \in(P)$,

(G2) $Y$ is symmetric and $Y \notin(P)$. 
Similarly, we will say that the property $P$ is good for the Copson construction if $1^{\circ}$ and $2^{\circ}$ above hold with $C$ in (G1) replaced with $C^{*}$. Moreover, the property $P$ is good if it is good for both the Cesàro and Copson constructions. The definitions given do not look particularly restrictive, but we will give some examples.

EXAMPLE 6.5. (a) Let us start by showing that order continuity is a good property. Take $P=\mathrm{OC}$. Obviously, order continuity is invariant under order isomorphism. Furthermore, if $X \in(\mathrm{OC})$, then $\left.X\right|_{A} \in(\mathrm{OC})$ for every $\emptyset \neq A \subset[0,1]$. It remains to find two nontrivial symmetric spaces on $[0,1]$ with properties (G1) and (G2). Note that if $X \in(\mathrm{OC})$, then $T X \in(\mathrm{OC})$ (see [56. Lemma 1(a)] and Corollary 6.1). Then, for example, we may take $X=L^{1}[0,1]$ and $Y$ any symmetric space on $[0,1]$ with $Y \neq Y_{a}$, or $Y=$ $L^{\infty}[0,1]$ and $X$ any order continuous symmetric space on $[0,1]$.

(b) We will show that the Dunford-Pettis property (DPP) is a good property (see [1, p. 115] for the definition and [26] for related results). Clearly, the Dunford-Pettis property is invariant under order isomorphism. Next, complemented subspaces of spaces with the Dunford-Pettis property also have it, so condition $2^{\circ}$ is obviously satisfied. Moreover, Kamińska-Mastyło [42] proved that there are exactly two nonisomorphic symmetric spaces on $[0,1]$ with the Dunford-Pettis property, namely $L^{1}[0,1]$ and $L^{\infty}[0,1]$. Therefore, if $X=L^{1}[0,1]$ and $Y$ is a reflexive symmetric space on $[0,1]$, then

$$
C L^{1}[0,1]=\operatorname{Ces}_{1}[0,1] \simeq L^{1}[0,1] \in(\mathrm{DPP}) \quad \text { and } \quad Y \notin(\mathrm{DPP}) .
$$

Moreover,

$$
C^{*} L^{1}[0,1]=\operatorname{Cop}_{1}[0,1] \simeq L^{1}[0,1] \in(\mathrm{DPP})
$$

(c) Let $p \geq 1$ and suppose that $X$ is a Banach function space on $I$ which is $p$-concave with constant $L \geq 1$ (see [57, pp. 45-46] for the definition) and such that the Cesàro operator $C$ is bounded on $X$. First, we will show that then also $C X$ is $p$-concave with constant $L$. Recall that $\left.L^{1}(I)\right|_{[0, x]}$ for $0<x \in I$ is 1 -convex with constant 1 and $p$-concave with constant 1 , that is,

$$
\begin{aligned}
\left(\sum_{k=1}^{n}\left\|f_{k}\right\|_{L^{1}(I) \mid[0, x]}^{p}\right)^{1 / p} & =\left(\sum_{k=1}^{n}\left(\int_{0}^{x}\left|f_{k}(t)\right| \mathrm{d} t\right)^{p}\right)^{1 / p} \\
& \leq \int_{0}^{x}\left(\sum_{k=1}^{n}\left|f_{k}(t)\right|^{p}\right)^{1 / p} \mathrm{~d} t=\left\|\left(\sum_{k=1}^{n}\left|f_{k}\right|^{p}\right)^{1 / p}\right\|_{\left.L^{1}(I)\right|_{[0, x]}}
\end{aligned}
$$

for every $0<x \in I$ (see [57, Proposition 1.d.5], [62, Theorem 4.3] and [12, 
second part of the proof of Theorem 4]). Therefore, we see immediately that

$$
\begin{aligned}
\left(\sum_{k=1}^{n}\left(C\left|f_{k}\right|\right)^{p}\right)^{1 / p} & =\left(\sum_{k=1}^{n}\left(\frac{1}{x} \int_{0}^{x}\left|f_{k}(t)\right| \mathrm{d} t\right)^{1 / p}\right)^{1 / p} \\
& \leq \frac{1}{x} \int_{0}^{x}\left(\sum_{k=1}^{n}\left|f_{k}(t)\right|^{p}\right)^{1 / p} \mathrm{~d} t=C\left(\sum_{k=1}^{n}\left|f_{k}\right|^{p}\right)^{1 / p} .
\end{aligned}
$$

Using the above inequality and the $p$-concavity of $X$, we obtain

$$
\begin{aligned}
\left(\sum_{k=1}^{n}\left\|f_{k}\right\|_{C X}^{p}\right)^{1 / p} & =\left(\sum_{k=1}^{n}\left\|C\left|f_{k}\right|\right\|_{X}^{p}\right)^{1 / p} \\
& \leq L\left\|\left(\sum_{k=1}^{n}\left(C\left|f_{k}\right|\right)^{p}\right)^{1 / p}\right\|_{X} \\
& \leq L\left\|C\left(\sum_{k=1}^{n}\left|f_{k}\right|^{p}\right)^{1 / p}\right\|_{X}=L\left\|\left(\sum_{k=1}^{n}\left|f_{k}\right|^{p}\right)^{1 / p}\right\|_{C X},
\end{aligned}
$$

for all $f_{1}, \ldots, f_{n} \in C X$. Consequently, also $C X$ is $p$-concave with the same constant as for $X$.

Now we claim that $p$-concavity is a good property. It is well known that this property is invariant under order isomorphism; this follows, for example, from the fact that if $X$ is $p$-concave and $S: Y \rightarrow X$ is a positive operator, and $Y$ is a Banach lattice, then $S$ is $p$-concave as well [57, 1.d.9, p. 55]. It is also clear that condition $2^{\circ}$ is satisfied. Taking, for example, $X=L^{p}[0,1]$ for $1<p<\infty$ and $Y=L^{\infty}[0,1]$ we conclude that $p$-concavity is a good property for the Cesàro construction.

To show that it is also good for the Copson construction we will prove first that if $X$ and $Y$ are $p$-concave Banach function spaces on $I$, then $X \cap Y$ is $p$-concave as well. Let $Z=X \cap Y$ and $\|f\|_{Z}=\max \left\{\|f\|_{X},\|f\|_{Y}\right\}$. Take $f_{1}, \ldots, f_{n} \in Z$ and denote by $L_{X}, L_{Y}>0$ the constants of $p$-concavity of $X$ and $Y$, respectively (cf. [57]). We have

$$
\left\|\left(\sum_{k=1}^{n}\left|f_{k}\right|^{p}\right)^{1 / p}\right\|_{Z} \geq\left\|\left(\sum_{k=1}^{n}\left|f_{k}\right|^{p}\right)^{1 / p}\right\|_{X} \geq \frac{1}{L_{X}}\left(\sum_{k=1}^{n}\left\|f_{k}\right\|_{X}^{p}\right)^{1 / p},
$$

and

$$
\left\|\left(\sum_{k=1}^{n}\left|f_{k}\right|^{p}\right)^{1 / p}\right\|\left\|_{Z} \geq\right\|\left(\sum_{k=1}^{n}\left|f_{k}\right|^{p}\right)^{1 / p} \|_{Y} \geq \frac{1}{L_{Y}}\left(\sum_{k=1}^{n}\left\|f_{k}\right\|_{Y}^{p}\right)^{1 / p} .
$$

Therefore, setting $L=\max \left\{L_{X}, L_{Y}\right\}$, we have

$$
\left\|\left(\sum_{k=1}^{n}\left|f_{k}\right|^{p}\right)^{1 / p}\right\|_{Z} \geq \frac{1}{2 L}\left(\left(\sum_{k=1}^{n}\left\|f_{k}\right\|_{X}^{p}\right)^{1 / p}+\left(\sum_{k=1}^{n}\left\|f_{k}\right\|_{Y}^{p}\right)^{1 / p}\right) .
$$


From the triangle inequality for $\ell^{p}$, we immediately obtain

$$
\begin{aligned}
\left\|\left(\sum_{k=1}^{n}\left|f_{k}\right|^{p}\right)^{1 / p}\right\|_{Z} & \geq \frac{1}{2 L}\left(\sum_{k=1}^{n}\left(\left\|f_{k}\right\|_{X}+\|f\|_{Y}\right)^{p}\right)^{1 / p} \\
& \geq \frac{1}{2 L}\left(\sum_{k=1}^{n}\left(\max \left\{\|f\|_{X},\|f\|_{Y}\right\}\right)^{p}\right)^{1 / p}=\frac{1}{2 L}\left(\sum_{k=1}^{n}\left\|f_{k}\right\|_{Z}^{p}\right)^{1 / p} .
\end{aligned}
$$

But this means that $Z$ is $p$-concave and the claim follows.

Now, it is clear that if $X$ is a $p$-concave symmetric space such that both $C$ and $C^{*}$ are bounded on $X$, then $C^{*} X$ is also $p$-concave. Indeed, suppose that $I=[0,1]$, because if $I=[0, \infty)$ there is nothing to prove (see (6.1)). Since $L^{1}[0,1]$ is $p$-concave with constant 1 , it follows from the first part that $C X[0,1]$ is also $p$-concave. Therefore, $C X[0,1] \cap L^{1}[0,1]$ is $p$-concave as well. But in view of 6.2 this means that also $C^{*} X[0,1]$ is $p$-concave. Finally, we may take the same spaces $X, Y$ as above for the Cesàro construction.

Theorem 6.6. Let $T=C$ or $T=C^{*}$. If $P$ is a good property, then

(i) $L^{1}[0,1]$ has the property $P$,

(ii) $P$ does not transfer from $T X$ into $X$, that is, there is a Banach function space $Z$ such that $T Z$ is nontrivial, $T Z \in(P)$ and $Z \notin(P)$.

Proof. (i) Take the Banach function space $X$ from the definition of good property. Applying Lemma 4.1 we conclude that there are $0<a<b<1$ such that $\left.C X\right|_{[a, b]}$ is order isomorphic to $\left.L^{1}\right|_{[a, b]}=L^{1}[a, b]$ which is order isomorphic to $L^{1}[0,1]$, since it is enough to take $\mathcal{H}: L^{1}[0,1] \rightarrow L^{1}[a, b]$ defined by

$$
\mathcal{H}: f(x) \mapsto \mathcal{H} f(x):= \begin{cases}f\left(\frac{1}{b-a} x-\frac{a}{b-a}\right) & \text { if } x \in(a, b), \\ 0 & \text { if } x \in[0,1] \backslash(a, b) .\end{cases}
$$

By the definition of good property we conclude that $\left.C X\right|_{[a, b]} \in(P)$ and consequently $L^{1}[0,1] \in(P)$.

(ii) In virtue of the definition of good property, take a symmetric Banach function space $Y$ on $[0,1]$ such that $Y \notin(P)$. Define $Z[0,1]:=L^{1}[0, a] \oplus$ $Y[a, b] \oplus L^{1}[b, 1]$. We claim that $Z \notin(P)$. Indeed, since $Y \notin(P)$, a dilation operator $\sigma_{\tau}$ is bounded in any symmetric space [14, p. 148] and a translation operator $T: f(x) \mapsto T f(x)=f(x-a)$ is also bounded in any symmetric space, so $Y[a, b] \notin(P)$ (by property $1^{\circ}$ of the definition of good property), whence $\left.Z\right|_{[a, b]} \notin(P)$. Thus, by property $2^{\circ}, Z \notin(P)$. Finally, we show that $T Z \in(P)$. Since $L^{\infty}[0,1] \hookrightarrow Y \hookrightarrow L^{1}[0,1]$, by Lemma 6.2 we conclude that $T Z=T\left(L^{1}[0,1]\right)$. If $T=C$, then $C\left(L^{1}[0,1]\right)$ is even isometric to $L^{1}[0,1]$ (see Example 3.3(b)). Thus by part (i), applying property $1^{\circ}, C Z \in(P)$. If $T=C^{*}$, it is enough to recall that $\mathrm{Cop}_{1} \equiv L^{1}$ (see Example 3.3(c)). 
Obviously, $T Z$ is nontrivial, because $L^{\infty}[0,1] \hookrightarrow Z$, whence $T\left(L^{\infty}[0,1]\right)$ $\hookrightarrow T Z$.

It seems interesting that when we restrict the class of spaces under consideration to symmetric spaces, it may happen that the above theorem is not true. For example, it was proved by Kiwerski-Tomaszewski [48, Theorem 3] that if $X$ is a symmetric space such that the Cesàro operator is bounded on $X$, then $X$ is order continuous if and only if $C X$ is (see also Corollary 6.1). Moreover, if $I=[0, \infty)$ and we allow $C X=\{0\}$, then it is easy to see that $C\left(L^{1} \cap L^{\infty}\right)=\{0\} \in(\mathrm{OC})$ but $L^{1} \cap L^{\infty} \notin(\mathrm{OC})$.

The results in the next section show that also fixed point properties do not transfer from $X$ to the Cesàro or Copson function spaces.

7. Applications to metric fixed point theory. A Banach space $X=$ $\left(X,\|\cdot\|_{X}\right)$ has the fixed point property $(X \in(\mathrm{FPP}))$ if every nonexpansive mapping $T: K \rightarrow K$, that is, the mapping satisfying

$$
\|T(x)-T(y)\| \leq\|x-y\| \quad \text { for all } x, y \in K
$$

on every nonempty, closed, bounded and convex subset $K$ of $X$, has a fixed point, i.e., there exists $x_{0} \in K$ such that $T\left(x_{0}\right)=x_{0}$. If the same holds for every nonempty, weakly compact and convex subset $K$ of $X$, we say that the space has the weak fixed point property (we write $X \in(\mathrm{wFPP})$ ). Of course, if $X$ has the fixed point property, then it has the weak fixed point property, and both properties are equivalent in the class of reflexive spaces. The spaces $c_{0}, \ell^{1}, L^{1}[0,1], L^{\infty}[0,1], L^{p, 1}[0, \infty)$ and $C[0,1]$ fail the fixed point property and the spaces $\ell^{\infty}, c_{0}(\Gamma)$ and $\ell^{1}(\Gamma)$, for $\Gamma$ uncountable, cannot even be renormed to have the fixed point property [31, Theorem 2, Corollary 3 and remark after Proposition 7]. However, $c_{0}$ and $\ell^{1}$ have the weak fixed point property but $L^{1}[0,1] \notin($ wFPP $)$, as proved by Alspach [3].

We are now ready to prove the main result of this section.

ThEOREM 7.1. Let $T=C$ or $T=C^{*}$. If $X$ is a Banach function space on $I$ such that $T X \neq\{0\}$, then $T X$ fails to have the fixed point property. Moreover,

(i) $(T X)^{*}$ cannot be renormed to have the fixed point property,

(ii) $(T X)^{*}$ fails to have the weak fixed point property.

Proof. That $C X \notin(\mathrm{FPP})$ follows immediately from Theorem 4.5 and Dowling-Lennard's result [29] that a Banach space which contains an asymptotically isometric copy of $\ell^{1}$ fails to have the fixed point property (see also [30. Theorem 2.3 and Corollary 2.11]). For $C^{*} X$ we apply Theorem 4.6.

(i) It is known that if a Banach space $X$ contains a complemented copy of $\ell^{1}$, then $X^{*}$ cannot be renormed to have the fixed point property [31, Corollary 4]. Thus it suffices to apply Corollary 4.3. 
(ii) Recall Dilworth-Girardi-Hagler's result [27, Theorem 2] that a Banach space $X$ contains an asymptotically isometric copy of $\ell^{1}$ if and only if $X^{*}$ contains an isometric copy of $L^{1}[0,1]$. Combining Theorem 4.5 with the Dilworth-Girardi-Hagler theorem we find that $(C X)^{*}$ contains an isometric copy of $L^{1}[0,1]$. In view of Alspach's result [3] this means that $(C X)^{*} \notin(\mathrm{wFPP})$. Again, in the case of $C^{*} X$ we simply use Theorem 4.6 .

From Alspach's result [3] and our Theorem 4.4 we easily obtain

Corollary 7.2. Let $T=C$ or $T=C^{*}$. Assume that $X$ is a Banach function space on $I$ with $T X \neq\{0\}$. Then there is an equivalent norm on $T X$ for which $T X$ fails to have the weak fixed point property.

In the next remark we collect some known results concerning the (weak) fixed point property and copies of $\ell^{\infty}$.

REMARK 7.3. Let $X$ be a Banach space.

(i) If $X$ contains an isomorphic copy of $\ell^{\infty}$, then $X$ cannot be renormed to have the fixed point property.

(ii) If $X$ contains an isometric copy of $\ell^{\infty}$, then $X$ fails to have the weak fixed point property.

Proof. (i) It follows from Pełczyński's result of [67] that a separable Banach space $X$ contains an isomorphic copy of $\ell^{1}$ if and only if $X^{*}$ contains an isomorphic copy of $\ell^{1}(\Gamma)$ for some uncountable set $\Gamma$. In particular, $\left(\ell^{1}\right)^{*}=\ell^{\infty}$ and consequently $\ell^{\infty}$ contains an isomorphic copy of $\ell^{1}(\Gamma)$. Moreover, by Dowling-Lennard-Turett's result [31, Theorem 1] any renorming of $\ell^{1}(\Gamma)$ contains an asymptotically isometric copy of $\ell^{1}$. But a Banach space which contains an asymptotically isometric copy of $\ell^{1}$ fails to have the fixed point property [30]. Therefore, so does $X$.

(ii) A classical result is that $\ell^{\infty}$ is the universal space for all separable Banach spaces, i.e., every separable Banach space $X$ can be isometrically embedded into $\ell^{\infty}$ (just take a dense subset $\left\{x_{n}: n \in \mathbb{N}\right\} \subset S(X)$ with $x_{n}^{*} x_{n}=1$ for all $n \in \mathbb{N}$, where $\left\{x_{n}^{*}: n \in \mathbb{N}\right\} \subset S\left(X^{*}\right)$, and put $T: X \ni x \mapsto$ $\left.\left(x_{n}^{*} x\right)_{n=1}^{\infty} \in \ell^{\infty}\right)$. Therefore, in particular, $\ell^{\infty}$ contains an isometric copy of $L^{1}[0,1]$. Again, by Alspach's result [3], $X \notin(\mathrm{wFPP})$.

Lozanovskiı [58] proved that a Banach function space $X$ is order continuous if and only if it contains no isomorphic copy of $\ell^{\infty}$. Moreover, if $X$ is a symmetric space and $C$ is bounded on $X$, then $C X$ is order continuous if and only if $X$ is (see [48]). Consequently, by Remark 7.3 and Corollary 6.1. we have

Corollary 7.4. Let $T=C$ or $T=C^{*}$. If $X$ is a symmetric space on $I$ such that $C$ and $C^{*}$ are bounded on $X$ and $X$ is not order continuous, then $T X$ cannot be renormed to have the fixed point property. 
Proposition 7.5. $\mathrm{Ces}_{1}[0,1], \mathrm{Ces}_{\infty}, \mathrm{Cop}_{1}, \mathrm{Cop}_{\infty}$ and nontrivial Tandori function spaces $\widetilde{X}$ fail to have the weak fixed point property.

Proof. Since $\operatorname{Ces}_{1}[0,1] \equiv L^{1}(\ln (1 / t))[0,1]$ (see Example $3.3(\mathrm{~b})$ ), and since $L^{1}(\ln (1 / t))[0,1]$ is isometric to $L^{1}[0,1]$ and $L^{1}[0,1] \notin$ (wFPP) by Alspach's result [3], it follows that $\mathrm{Ces}_{1}[0,1] \notin(\mathrm{wFPP})$.

Arguing in the same way, we infer that $\operatorname{Cop}_{1} \equiv L^{1}$ and $\operatorname{Cop}_{\infty} \equiv L^{1}(1 / t)$ (see Example 3.3.(c)) also fail to have the weak fixed point property.

If $\widetilde{X} \neq\{0\}$, then the claim follows from Proposition 4.8 and Remark 7.3 For $\mathrm{Ces}_{\infty}$ we apply Proposition 4.9 and Remark 7.3 , respectively.

8. Generalizations and applications. Until now, most of the results we have obtained for the Cesàro and Copson function spaces have been proven in the class of Banach function spaces and under the nontriviality assumption. It turns out that we can transfer (without much effort) the most important results from Sections 4 and 7 to even more general optimal domains. We start with some definitions.

Denote by $\mathcal{H}_{w}$ the weighted Cesàro operator, defined as

$$
\mathcal{H}_{w}: f \mapsto \mathcal{H}_{w} f(x):=w(x) \int_{0}^{x} f(t) \mathrm{d} t \quad \text { for } t \in I,
$$

where $w$ is a positive weight on $I$. For a Banach function space $X$ on $I$ the weighted Cesàro function space $C_{w} X(I)=C_{w} X$ is

$$
C_{w} X:=\left\{f \in L^{0}: \mathcal{H}_{w}|f| \in X\right\} \quad \text { with the norm }\|f\|_{C_{w} X}=\left\|\mathcal{H}_{w}|f|\right\|_{X} .
$$

For $X=L^{p}$, where $1 \leq p<\infty$, these spaces were studied by KamińskaKubiak [41] and Kubiak [52]. Observe that the study of the spaces $C_{p, w}:=$ $\mathcal{H}_{w} L^{p}$ is more or less equivalent to the study of $C L^{p}(w)$, that is, the Cesàro operator on weighted $L^{p}$-spaces. Of course, if we take $w(x)=1 / x$ then $C_{w} X \equiv C X$. Moreover, if $w \equiv 1$ then $\mathcal{H}_{w}=V$, where $V$ denotes the Volterra operator

$$
V: f \mapsto V f(x):=\int_{0}^{x} f(t) \mathrm{d} t \quad \text { for } t \in I .
$$

Easy computations involving Fubini's theorem show that the conjugate operator $\mathcal{H}_{w}^{*}$ to the weighted Cesàro operator $\mathcal{H}_{w}$ is given by the formula

$$
\mathcal{H}_{w}^{*}: f \mapsto \mathcal{H}_{w}^{*} f(x):=\int_{I \cap[x, \infty)} w(t) f(t) \mathrm{d} t \quad \text { for } t \in I .
$$

The space $C_{w}^{*} X(I)=C_{w}^{*} X$ associated with this operator can be called the weighted Copson function space. Again, if $w(t)=1 / t$ then $C_{w}^{*} X \equiv C^{*} X$ and if $w(t) \equiv 1$ then $C_{w}^{*} X \equiv V^{*} X$. 
Note that

$$
\|f\|_{C_{w} X}=\left\|w(x) \int_{0}^{x}|f(t)| \mathrm{d} t\right\|_{X}=\|C|f|\|_{X(v)}=\|f\|_{C Y},
$$

that is, $C_{w} X \equiv C Y$, where $Y=X(v)$ and $v(x):=x w(x)$. That is why it is easy to transfer claims about the Cesàro spaces $C X$ for $X$ being a Banach function space (more often nonsymmetric) to the spaces $C_{w} X$. Moreover, $C_{w} X \equiv V(X(w))$.

It is easy to see that $C_{w} X$ is nontrivial if and only if $w(x) \chi_{\left[\lambda_{0}, m(I)\right)}(x) \in X$ for some $0<\lambda_{0}<m(I)$ (cf. (8.1) and [54, Theorem 1(a,b)]). Furthermore, if $C_{w}^{*} X$ is nontrivial then $\chi_{\left[0, \lambda_{0}\right]} \in X$ for some $0<\lambda_{0}<m(I)$ (cf. Lemma 3.1). Keeping in mind this observation and following the proofs of Lemmas 4.1 and 4.2 we can show

Lemma 8.1. Let $X$ be a Banach function space on $I$.

(i) Assume that $C_{w} X \neq\{0\}$. Then there exist $0 \leq \lambda_{0} \in I$ with

$$
\left\|w(x) \chi_{[b, m(I))}(x)\right\|_{X}\|f\|_{L^{1}} \leq\|f\|_{C_{w} X} \leq\left\|w(x) \chi_{[a, m(I))}(x)\right\|_{X}\|f\|_{L^{1}}
$$

for all $f \in C_{w} X$ such that $\operatorname{supp}(f) \subset[a, b]$, where $0 \leq \lambda_{0}<a<b<m(I)$.

(ii) If $C_{w}^{*} X \neq\{0\}$, then we can find $0<\eta_{0} \in I$ with

$$
\left\|\chi_{[0, a]}\right\|_{X}\|f\|_{L^{1}(w)} \leq\|f\|_{C_{w}^{*} X} \leq\left\|\chi_{[0, b]}\right\|_{X}\|f\|_{L^{1}(w)}
$$

for all $f \in C_{w}^{*} X$ such that $\operatorname{supp}(f) \subset[a, b]$, where $0<a<b<\eta_{0} \leq m(I)$.

The next theorem, in the case of the weighted Cesàro function space $C_{w} X$, easily follows from the identification $C_{w} X \equiv C Y$, where $Y=X(v)$ and $v(x)=x w(x)$ (cf. 8.1) ), and Theorem 4.5. On the other hand, if $T=\mathcal{H}_{w}^{*}$, it is sufficient to use the same argument as in the proof of Theorem 4.6 and Lemma 8.1(ii) instead of Lemma 4.2 (actually, the proof will be almost identical, because we can use the same function $G_{X}$ ). Summarizing the above discussion, we can obtain

THeOREM 8.2. Let $X$ be a Banach function space on $I$ and $T=\mathcal{H}_{w}$ or $T=\mathcal{H}_{w}^{*}$. Then the nontrivial space $T X$ contains an order asymptotically isometric copy of $\ell^{1}$.

A similar result for $C_{p, w}:=C_{w} L^{p}$, where $1 \leq p<\infty$, was obtained by Kubiak [52, Theorem 5.1].

Now, a direct consequence of Theorem 8.2 and Dowling-Lenard-Turett's result [30] (cf. proof of Theorem 7.1) is

THEOREM 8.3. Let $X$ be a Banach function space on $I$ and $T=\mathcal{H}_{w}$ or $T=\mathcal{H}_{w}^{*}$. Then $T X$ fails to have the fixed point property whenever it is nontrivial.

A direct application of the above discussion yields 
COROLlary 8.4. The nontrivial Volterra space VX contains an asymptotically isometric copy of $\ell^{1}$, and so fails to have the fixed point property.

Finally, let us mention that $\mathrm{Vol}_{1}:=V L^{1} \equiv L^{1}(1-t)$ and $\mathrm{Vol}_{\infty}:=$ $V L^{\infty} \equiv L^{1}$ (cf. Example 3.3 (b, c)) and consequently we have

TheOREM 8.5. The spaces $\mathrm{Vol}_{1}$ and $\mathrm{Vol}_{\infty}$ fail to have the weak fixed point property.

9. Appendix. In the proof of Theorem 4.5 it was necessary for the function $F_{X}$ to be continuous at least at one point. It turned out that it is always continuous at uncountably many points. However, the question whether this function is actually continuous on the whole domain may be of interest.

Lemma 9.1. Let $X$ be a Banach function space on I such that the operator $C$ is bounded on $X$. Assume that one of the following holds true:

(i) $X$ is order continuous,

(ii) $X$ is symmetric and $X \hookrightarrow L^{\infty}$.

Then the function $F_{X}$ is finitely valued and continuous for all $0<\lambda \in I$.

Proof. Actually, the proof of Lemma 4.1 shows that $F_{X}$ is finitely valued. Moreover, $\operatorname{supp}(C X)=I$ because the Cesàro operator is bounded on $X$, by Theorem D. It remains to prove that $F_{X}$ is also continuous. We will consider two situations.

Assume that $I=[0,1]$. Fix $0<\lambda_{0}<1$ and take a sequence $\left(\lambda_{n}\right)_{n=1}^{\infty} \subset[0,1]$ such that $\lambda_{n} \rightarrow \lambda_{0}$. We will show that

$$
F_{X}\left(\lambda_{n}\right) \rightarrow F_{X}\left(\lambda_{0}\right)
$$

that is, $F_{X}$ is continuous on $(0,1)$. Note first that there exist $0<\varepsilon<$ $\min \left\{\lambda_{0}, 1-\lambda_{0}\right\}$ and $N \in \mathbb{N}$ such that

$$
\begin{aligned}
0 & \leq\left|f_{\lambda_{n}}(x)-f_{\lambda_{0}}(x)\right| \\
& =\frac{1}{x} \chi_{\left(\min \left\{\lambda_{0}, \lambda_{n}\right\}, \max \left\{\lambda_{0}, \lambda_{n}\right\}\right)}(x) \leq \max _{n \geq N}\left\{\frac{1}{\lambda_{0}}, \frac{1}{\lambda_{n}}\right\} \chi_{\left(\lambda_{0}-\varepsilon, \lambda_{0}+\varepsilon\right)}(x)
\end{aligned}
$$

for $n \geq N$ and $0<x \leq 1$. Put

$$
h_{n}:=\frac{1}{x} \chi_{\left(\min \left\{\lambda_{0}, \lambda_{n}\right\}, \max \left\{\lambda_{0}, \lambda_{n}\right\}\right)} \quad \text { and } \quad H:=\max _{n \geq N}\left\{\frac{1}{\lambda_{0}}, \frac{1}{\lambda_{n}}\right\} \chi_{\left(\lambda_{0}-\varepsilon, \lambda_{0}+\varepsilon\right)} .
$$

Then $h_{n} \rightarrow 0$ almost everywhere on $[0,1]$ as $n \rightarrow \infty$, and it follows from 9.2 that $0 \leq h_{n} \leq H$. We claim that $H \in X_{a}$. In fact, if $X$ is a symmetric space and $X \hookrightarrow L^{\infty}$, then $X_{a}=X_{b}$, where $X_{b}$ is the closure in $X$ of the set of bounded functions supported in sets of finite measure (see, for example, [48, Theorem $\mathrm{B}]$ ). It is clear that $H$ is such a function and so $H \in X_{a}$. However, if $X$ is an order continuous Banach function space then the situation is a 
little different. Due to boundedness of the Cesàro operator and Theorem D, we can see that $\chi_{[\lambda, 1]} \in X$ for all $0<\lambda<1$. Therefore, $H \in X=X_{a}$ and the claim follows. Just from the definition of order continuity and $(9.2)$ we obtain

$$
\begin{aligned}
0 \leq\left|F_{X}\left(\lambda_{n}\right)-F_{X}\left(\lambda_{0}\right)\right| & =\|\| \frac{1}{x} \chi_{\left(\lambda_{n}, 1\right]}(x)\left\|_{X}-\right\| \frac{1}{x} \chi_{\left(\lambda_{0}, 1\right]}(x) \|_{X} \mid \\
& \leq\left\|\frac{1}{x} \chi_{\left(\lambda_{n}, 1\right]}(x)-\frac{1}{x} \chi_{\left(\lambda_{0}, 1\right]}(x)\right\|_{X} \\
& =\left\|\frac{1}{x} \chi_{\left(\min \left\{\lambda_{0}, \lambda_{n}\right\}, \max \left\{\lambda_{0}, \lambda_{n}\right\}\right)}(x)\right\|_{X}=\left\|h_{n}\right\|_{X} \rightarrow 0
\end{aligned}
$$

as $n \rightarrow \infty$. This proves 9.1). In the remaining case of $\lambda_{0}=1$, the argument is essentially the same, so we omit it.

Now suppose that $I=[0, \infty)$. Note only that in this case $\frac{1}{x} \chi_{[\lambda, \infty)}(x) \in X$ for each $0<\lambda \in I$ and $\chi_{[a, b]} \in X$ for each $0<a<b<\infty$, by Theorem D. Thus we can proceed as in the previous case.

It is not surprising that we can also prove an analogous lemma for the function $G_{X}$.

Lemma 9.2. Let $X$ be a Banach function space on I such that the Copson operator is bounded on $X$. Assume that one of the following holds true:

(i) $X$ is order continuous,

(ii) $X$ is symmetric and $X \hookrightarrow L^{\infty}$.

Then the function $G_{X}$ is finitely valued and continuous for all $\lambda \in I$.

Proof. We proceed as in the proof of Lemma 9.1 and use Corollary 3.2 instead of the proof of Lemma 4.2 .

If we replace the assumption that the operator $T$, where $T=C$ or $T=C^{*}$, is bounded on $X$ by the assumption that $T X \neq\{0\}$, then using the same arguments as before we find that $F_{X}$ (resp. $G_{X}$ ) is finitely valued and continuous for all $x \in \operatorname{int}(\operatorname{supp}(T X))$.

The above lemma does not exclude the possibility that $F_{X}$ is continuous at each $x$ with $0<x \in I$ but $X$ has trivial order continuous part. In fact, it is rather common for spaces with $X_{a}$ trivial to have this property. We will now give some examples illustrating the discussion about the continuity of $F_{X}$.

EXAMPle 9.3. (a) Let $X$ be a Banach function space on $I$ and $w_{0}, w_{1}: I \rightarrow(0, \infty)$ be weights that differ only on a set of measure zero, i.e., $m\left(\left\{x \in I: w_{0}(x) \neq w_{1}(x)\right\}\right)=0$. Then, of course, $X\left(w_{0}\right) \equiv X\left(w_{1}\right)$. In particular, if $X$ satisfies the assumptions of Lemma 9.1 and $w_{1}=\mathfrak{D}$, where

$$
\mathfrak{D}: I \ni x \mapsto \mathfrak{D}(x):=\chi_{I \backslash \mathbb{Q}}(x)
$$


is the Dirichlet function, then $X \equiv X(\mathfrak{D})$ and $\mathfrak{D}$ is a nowhere continuous function on $I$ but $F_{X(\mathfrak{D})}$ is a continuous function for all $0<x \in I$.

(b) Put

$$
w_{2}:[0,1] \ni x \mapsto w_{2}(x):=2 \chi_{\mathfrak{C}}(x)+\chi_{[0,1] \backslash \mathfrak{C}}(x),
$$

where $\mathfrak{C}$ is the Smith-Volterra-Cantor set (or the fat Cantor set). The set of discontinuities of $w_{2}$ is $\mathfrak{C}$, so it is uncountable and of positive measure. However, the set of discontinuities of $F_{Y}$, where $Y:=L^{\infty}\left(w_{2}\right)[0,1]$, is at most countable.

(c) Let $\left(q_{n}\right) \subset \mathbb{Q} \cap[0,1]$ and put $Z:=L^{\infty}\left(w_{3}\right)[0,1]$, where

$$
w_{3}:[0,1] \ni x \mapsto w_{3}(x):=\sum_{\substack{q_{n}<x \\ q_{n} \in \mathbb{Q} \cap[0,1]}}^{\infty} 2^{-n} .
$$

Then $F_{Z}$ is discontinuous at every rational number from the interval $[0,1]$ and continuous elsewhere.

(d) Let $X$ be a symmetric space such that $X \cong L^{\infty}$, i.e., $X$ and $L^{\infty}$ have the same elements and $\|f\|_{X}=A\|f\|_{L^{\infty}}$ for $f \in X$ and some constant $A>0$. Then, with the same notation as in the proof of Lemma 9.1, we have

$$
\begin{aligned}
\left|F_{X}\left(\lambda_{n}\right)-F_{X}\left(\lambda_{0}\right)\right| & =\left|\left\|\frac{1}{x} \chi_{\left(\lambda_{n}, m(I)\right)}(x)\right\|_{X}-\left\|\frac{1}{x} \chi_{\left(\lambda_{0}, m(I)\right)}(x)\right\|_{X}\right| \\
& =A\left|\frac{1}{\lambda_{n}}-\frac{1}{\lambda_{0}}\right| \rightarrow 0
\end{aligned}
$$

as $n \rightarrow \infty$. But this means that $F_{X}$ is continuous at $0<x \in I$. It is also worth noting that if $X$ is a symmetric space on $[0,1]$ then the condition $X_{a}=\{0\}$ is equivalent to $X=L^{\infty}[0,1]$ [48, Theorem B]. However, in the class of Orlicz spaces the condition $\left(L^{\Phi}\right)_{a}=\{0\}$ (that is, the Orlicz function $\Phi$ takes also infinite values) is equivalent to $L^{\Phi} \cong L^{\infty}[0,1]$. Moreover, for symmetric spaces on $[0, \infty)$ the condition $X_{a}=\{0\}$ is equivalent to $X \hookrightarrow L^{\infty}[0, \infty)$ (see also [48, Theorem B]).

(e) Let $Y$ be a Banach function space on $I$ such that $Y \cong X \cap L^{\infty}$, where

$$
X \cap L^{\infty}:=\left\{f \in L^{0}:\|f\|_{Y}:=\max \left\{\|f\|_{X},\|f\|_{L^{\infty}}\right\}<\infty\right\},
$$

and $X$ is an order continuous Banach function space on $I$. Of course, $Y_{a}=\{0\}$ but we can prove that $F_{Y}$ is continuous at $0<x \in I$ (we will sketch the proof only for $I=[0,1]$ because the remaining case is the same). Indeed, we have

$$
\begin{aligned}
\left|F_{Y}\left(\lambda_{n}\right)-F_{Y}\left(\lambda_{0}\right)\right| & =\left|\left\|\frac{1}{x} \chi_{\left(\lambda_{n}, 1\right]}(x)\right\|_{Y}-\left\|\frac{1}{x} \chi_{\left(\lambda_{0}, 1\right]}(x)\right\|_{Y}\right| \\
& =B\left|\left\|\frac{1}{x} \chi_{\left(\lambda_{n}, 1\right]}(x)\right\|_{X \cap L^{\infty}}-\left\|\frac{1}{x} \chi_{\left(\lambda_{0}, 1\right]}(x)\right\|_{X \cap L^{\infty}}\right|,
\end{aligned}
$$


for some constant $B>0$. Example 9.3(d) above and Lemma 9.1 imply that $F_{L^{\infty}}$ and $F_{X}$ are continuous at all $0<x \in I$. Consequently, $F_{Y}=$ $B \max \left\{F_{X}, F_{L^{\infty}}\right\}$ is also continuous at $0<x \in I$ as the maximum of two continuous functions, and the claim follows.

The same examples can be considered also in the context of the function $G_{X}$.

Acknowledgements. The first and second authors are supported by the Ministry of Science and Higher Education of Poland, grant number 0213/SBAD/0114 .

\section{References}

[1] F. Albiac and N. J. Kalton, Topics in Banach Space Theory, Springer, New York, 2006.

[2] A. Alexiewicz, On Cauchy's condensation theorem, Studia Math. 16 (1957), 80-85.

[3] D. E. Alspach, A fixed point free nonexpansive map, Proc. Amer. Math. Soc. 82 (1981), 423-424.

[4] S. V. Astashkin, On the geometric properties of Cesàro spaces, Mat. Sb. 203 (2012), no. 4, 61-80 (in Russian); English transl.: Sb. Math. 203 (2012), 514-533.

[5] S. V. Astashkin, K. Leśnik and M. Maligranda, Isomorphic structure of Cesàro and Tandori spaces, Canad. J. Math. 71 (2019), 501-532.

[6] S. V. Astashkin and L. Maligranda, Cesàro function spaces fail the fixed point property, Proc. Amer. Math. Soc. 136 (2008), 4289-4294.

[7] S. V. Astashkin and L. Maligranda, Structure of Cesàro function spaces, Indag. Math. (N.S.) 20 (2009), 329-379.

[8] S. V. Astashkin and L. Maligranda, Rademacher functions in Cesàro type spaces, Studia Math. 198 (2010), 235-247.

[9] S. V. Astashkin and L. Maligranda, Interpolation of Cesàro sequence and function spaces, Studia Math. 215 (2013), 39-69.

[10] S. V. Astashkin and L. Maligranda, A short proof of some recent results related to Cesàro function spaces, Indag. Math. (N.S.) 24 (2013), 589-592.

[11] S. V. Astashkin and L. Maligranda, Interpolation of Cesàro and Copson spaces, in: M. Kato et al. (eds.), Banach and Function Spaces IV (Kitakyushu, 2009), Yokohama Publ., Yokohama, 2014, 123-133.

[12] S. V. Astashkin and L. Maligranda, Structure of Cesàro function spaces: a survey, in: Banach Center Publ. 102, Inst. Math., Polish Acad. Sci., 2014, 13-40.

[13] G. Bennett, Factorizing classical inequalities, Mem. Amer. Math. Soc. 120 (1996), no. 576,138 pp.

[14] C. Bennett and R. Sharpley, Interpolation of Operators, Academic Press, Boston, 1988.

[15] C. Bessaga and A. Pełczyński, On extreme points in separable conjugate spaces, Israel J. Math. 4 (1966), 262-264.

[16] D. W. Boyd, Indices of function spaces and their relationship to interpolation, Canad. J. Math. 21 (1969), 1245-1254.

[17] M. Ciesielski, P. Kolwicz and A. Panfil, Local monotonicity structure of symmetric spaces with application, J. Math. Anal. Appl. 409 (2014), 649-662. 
[18] Y. Cui and H. Hudzik, Some geometric properties related to fixed point theory in Cesàro sequence spaces, Collect. Math. 50 (1999), 277-288.

[19] Y. Cui, H. Hudzik and Y. Li, On the Garcia-Falset coefficient in some Banach sequence spaces, in: Function Spaces (Poznań, 1998), Lecture Notes in Pure Appl. Math. 213, Dekker, New York, 2000, 141-148.

[20] Y. Cui, C. Meng and R. Płuciennik, Banach-Saks property and property $(\beta)$ in Cesàro sequence spaces, Southeast Asian Bull. Math. 24 (2000), 201-210.

[21] G. P. Curbera and W. J. Ricker, Banach lattices with the Fatou property and optimal domains of kernel operators, Indag. Math. (N.S.) 17 (2006), 187-204.

[22] G. P. Curbera and W. J. Ricker, Abstract Cesàro spaces: Integral representation, J. Math. Anal. Appl. 441 (2016), 25-44.

[23] G. P. Curbera and W. J. Ricker, On the Radon-Nikodym property in function spaces, Proc. Amer. Math. Soc. 145 (2017), 617-626.

[24] G. P. Curbera and W. J. Ricker, The weak Banach-Saks property for function spaces, Rev. R. Acad. Ciencias Exactas Fís. Nat. Ser. A Mat. 111 (2017), 657-671.

[25] O. Delgado and J. Soria, Optimal domain for the Hardy operator, J. Funct. Anal. 244 (2007), 119-133.

[26] J. Diestel, A survey of results related to the Dunford-Pettis property, Contemp. Math. 2, Amer. Math. Soc., 1980, 15-60.

[27] S. Dilworth, M. Girardi and J. Hagler, Dual Banach spaces which contain an isometric copy of $L^{1}$, Bull. Polish Acad. Sci. Math. 48 (2000), 1-12.

[28] P. N. Dowling, W. B. Johnson, C. J. Lennard and B. Turett, The optimality of James's distortion theorems, Proc. Amer. Math. Soc. 125 (1997), 167-174.

[29] P. N. Dowling and C. J. Lennard, Every nonreflexive subspace of $L^{1}[0,1]$ fails the fixed point property, Proc. Amer. Math. Soc. 125 (1997), 443-446.

[30] P. N. Dowling, C. J. Lennard and B. Turett, Renormings of $l^{1}$ and $c_{0}$ and fixed point properties, in: Handbook of Metric Fixed Point Theory, Kluwer, Dordrecht, 2001, 269-297.

[31] P. N. Dowling, C. J. Lennard and B. Turett, Reflexivity and the fixed-point property for nonexpansive maps, J. Math. Anal. Appl. 200 (1996), 653-662.

[32] P. N. Dowling, N. Randrianantoanina and B. Turett, Remarks on James's distortion theorems, Bull. Austral. Math. Soc. 57 (1998), 49-54.

[33] P. Foralewski, K. Leśnik and L. Maligranda, Some remarks on the level functions and their applications, Comment. Math. 56 (2016), 55-86.

[34] K. Goebel and W. A. Kirk, Topics in Metric Fixed Point Theory, Cambridge Univ. Press, 1990.

[35] G. H. Hardy, Notes on some points in the integral calculus, LX. An inequality between integrals, Messenger of Math. 54 (1925), 150-156.

[36] G. H. Hardy, J. E. Littlewood and G. Pólya, Inequalities, Cambridge Univ. Press, 1952.

[37] B. D. Hassard and D. A. Hussein, On Cesàro function spaces, Tamkang J. Math. 4 (1973), 19-25.

[38] H. Hudzik, Banach lattices with order isometric copies of $l^{\infty}$, Indag. Math. (N.S.) 9 (1998), 521-527.

[39] A. A. Jagers, A note on Cesàro sequence spaces, Nieuw Arch. Wisk. 22 (1974), $113-124$.

[40] A. Kamińska and D. Kubiak, On isometric copies of $\ell_{\infty}$ and James constants in Cesàro-Orlicz sequence spaces, J. Math. Anal. Appl. 349 (2009), 574-584.

[41] A. Kamińska and D. Kubiak, On the dual of Cesàro function spaces, Nonlinear Anal. 372 (2012), 2760-2773. 
[42] A. Kamińska and M. Mastyło, The Dunford-Pettis property for symmetric spaces, Canad. J. Math. 52 (2000), 789-803.

[43] L. V. Kantorovich and G. P. Akilov, Functional Analysis, Nauka, Moscow, 1977 (in Russian); English transl.: Pergamon Press, New York, 1982.

[44] W. Kirk and B. Sims, Handbook of Metric Fixed Point Property, Kluwer, Dordrecht, 2001.

[45] T. Kiwerski and P. Kolwicz, Isomorphic copies of $l^{\infty}$ in Cesàro-Orlicz function spaces, Positivity 21 (2017), 1015-1030.

[46] T. Kiwerski and P. Kolwicz, Rotundity and monotonicity properties of selected Cesàro function spaces, Positivity 22 (2018), 357-377.

[47] T. Kiwerski and P. Kolwicz, Isometric copies of of $l^{\infty}$ in Cesàro-Orlicz function spaces, Results Math. 73 (2018), 1-21.

[48] T. Kiwerski and J. Tomaszewski, Local approach to order continuity in Cesàro function spaces, J. Math. Anal. Appl. 455 (2017), 1636-1654.

[49] P. Kolwicz, K. Leśnik and L. Maligranda, Symmetrization, factorization and arithmetic of quasi-Banach function spaces, J. Math. Anal. Appl. 470 (2019), 1136-1166.

[50] B. I. Korenblyum, S. G. Kreĭn and B. Ya. Levin, On certain nonlinear questions of the theory of singular integrals, Dokl. Akad. Nauk SSSR (N.S.) 62 (1948), 17-20 (in Russian).

[51] S. G. Kreĭn, Yu. I. Petunin and E. M. Semenov, Interpolation of Linear Operators, Nauka, Moscow 1978 (in Russian); English transl.: Amer. Math. Soc., Providence, RI, 1982.

[52] D. Kubiak, Some geometric properties of the Cesàro function spaces, J. Convex Anal. 21 (2014), 189-200.

[53] A. Kufner, L. Maligranda and L. E. Persson, The Hardy Inequality. About its History and Some Related Results, Vydavatelsky Servis, Plzeň, 2007.

[54] K. Leśnik and M. Maligranda, On abstract Cesàro spaces. Duality, J. Math. Anal. Appl. 424 (2015), 932-951.

[55] K. Leśnik and M. Maligranda, On abstract Cesàro spaces. Optimal range, Integral Equations Operator Theory 81 (2015), 227-235.

[56] K. Leśnik and M. Maligranda, Interpolation of abstract Cesàro, Copson and Tandori spaces, Indag. Math. (N.S.) 27 (2016), 764-785.

[57] J. Lindenstrauss and L. Tzafriri, Classical Banach Spaces II. Function Spaces, Springer, Berlin, 1979.

[58] G. Ja. Lozanovskiǔ, On isomorphic Banach structures, Sibirsk. Mat. Zh. 10 (1969) 93-98 (in Russian); English transl.: Siberian Math. J. 10 (1969), 64-68.

[59] W. A. J. Luxemburg and A. C. Zaanen, Some examples of normed Köthe spaces, Math. Ann. 162 (1966), 337-350.

[60] L. Maligranda, Indices and interpolation, Dissertationes Math. (Rozprawy Mat.) 234 (1985), 49 pp.

[61] L. Maligranda, Orlicz Spaces and Interpolation, Sem. Mat. 5, Univ. Estadual de Campinas, Dep. Mat., Campinas, 1989.

[62] L. Maligranda, Type, cotype and convexity properties of quasi-Banach spaces, in: M. Kato and L. Maligranda (eds.), Banach and Function Spaces (Kitakyushu, 2003), Yokohama Publ., Yokohama, 2004, 83-120.

[63] P. Meyer-Nieberg, Banach Lattices, Springer, Berlin, 1991.

[64] A. Nekvinda and L. Pick, Optimal estimates for the Hardy averaging operator, Math. Nachr. 283 (2010), 262-271.

[65] S. Okada, W. J. Ricker and E. Sánchez Pérez, Optimal Domain and Integral Extension of Operators Acting in Function Spaces, Birkhäuser, Basel, 2008. 
[66] A. Pełczyński, On the isomorphism of the spaces $m$ and $M$, Bull. Acad. Polon. Sci. Sér. Sci. Math. Astronom. Phys. 6 (1958), 695-696.

[67] A. Pełczyński, On Banach spaces containing $L_{1}[0,1]$, Studia Math. 30 (1968), 231246.

[68] W. J. Ricker, On the optimally defined Hardy operator in $L^{p}$-spaces, Proc. Amer. Math. Soc. 146 (2018), 4693-4705.

[69] J. S. Shiue, A note on Cesàro function spaces, Tamkang J. Math. 1 (1970), 91-95.

[70] G. Sinnamon, Interpolation of spaces defined by the level function, in: Harmonic Analysis (Sendai, 1990), Springer, Tokyo, 1991, 190-193.

[71] G. Sinnamon, Spaces defined by the level function and their duals, Studia Math. 111 (1994), 19-52.

[72] G. Sinnamon, The level functions in rearrangement invariant spaces, Publ. Mat. 45 (2001), 175-198.

[73] G. Sinnamon, Transferring monotonicity in weighted norm inequalities, Collect. Math. 54 (2003), 181-216.

[74] G. Sinnamon, Monotonicity in Banach function spaces, in: Nonlinear Analysis, Function Spaces and Applications (NASFA 8), Vol. 8, Czech. Acad. Sci., Prague, 2007, 204-240.

[75] P. W. Sy, W. Y. Zhang and P. Y. Lee, The dual of Cesàro function spaces, Glas. Mat. Ser. III 22 (1987), 103-112.

[76] K. Tandori, Über einen speziellen Banachschen Raum, Publ. Math. Debrecen 3 (1954), 263-268.

[77] W. Wnuk, Banach Lattices with Order Continuous Norms, PWN, Warszawa, 1999.

[78] A. C. Zaanen, Riesz Spaces II, North-Holland, Amsterdam, 1983.

Tomasz Kiwerski, Paweł Kolwicz

Institute of Mathematics

Poznan University of Technology

Piotrowo $3 \mathrm{~A}$

60-965 Poznań, Poland

E-mail: tomasz.kiwerski@gmail.com

pawel.kolwicz@put.poznan.pl

Lech Maligranda

Department of Engineering Sciences and Mathematics

Luleå University of Technology

SE-971 87 Luleå, Sweden

E-mail: lech.maligranda@ltu.se

Current address:

Institute of Mathematics

Poznan University of Technology

Piotrowo 3A

60-965 Poznań, Poland

E-mail: lech.maligranda@put.poznan.pl 
